\title{
„[H]aving your own place $[\ldots]$ gives you all the control, you know? \\ Ergebnisse einer Kurzstudie zu den \\ Unterbringungssituationen queerer Geflüchteter ${ }^{1}$
}

Vanessa Einbrodt und Wael Mahmoud

\section{Einleitung}

"Firstly, I can say, in almost every [...] counselling or group meeting, the topic of living comes up. Because I think for every person who applies for asylum and comes in this system of Unterkunft [...] it is a big essential topic." (Interview mit Masha, LesMigraS)

Die vermehrte Ankunft von Geflüchteten in der Europäischen Union und Deutschland von 2014 bis 2017 (insbesondere von Juli 2015 bis Februar 2016) (Grote/BAMF 2018: 5) bei einer gleichzeitig mangelhaften Vorbereitung von entsprechenden Unterbringungsmöglichkeiten durch staatliche Stellen, führte vielerorts in Deutschland zu einer Überbelegung bereits

1 Die Kurzstudie mit dem Titel „Zufluchtsorte? Eine Kurzstudie zur Unterbringungssituation von LGBTIQ* Geflüchteten in Berlin“ wurde unter Leitung von Dr. Judith Vey im Rahmen des Projekts „Handlungsfähigkeit in der bundesdeutschen Unterbringung von Flüchtenden" am Zentrum Technik und Gesellschaft der TU Berlin durchgeführt. Ein besonderer Dank gilt unseren geflüchteten Gesprächspartner*innen sowie unseren Gesprächspartner*innen Masha von LesMigraS, Mahmoud Hassino von der Schwulenberatung und dem*der Interviewpartner*in von GLADT, die ihre Erfahrungen, ihr Wissen und ihre Expertise mit uns geteilt haben und ohne die dieses Projekt nicht möglich gewesen wäre. Ein weiterer Dank geht an Sabrina Apicella, Jan Drunkenmölle und Judith Vey für ihre hilfreichen Kommentare, Änderungsvorschläge und Überarbreitungen dieses Textes. Darüber hinaus bedanken wir uns herzlich bei der Magnus-Hirschfeld-Stiftung, die die vorliegende Kurzstudie durch ihre Förderung ermöglicht hat. Das Zitat stammt aus dem Interview mit der*dem Gesprächspartner*in Nur. Die meisten Interviews führten Wael Mahmoud als arabischer Erstsprachler und Vanessa Einbrodt als deutsche Erstsprachlerin gemeinsam auf Englisch bzw. auf Arabisch oder Russisch mit Übersetzung ins Englische. Daher sind die meisten der im Artikel verwendeten Zitate in englischer Sprache. Die Namen der interviewten Geflüchteten wurden verändert, um ihre Anonymität zu gewährleisten. Direkte Zitate wurden zur Verbesserung der Leserlichkeit sprachlich geglättet. 
existierender Geflüchtetenunterkünfte bzw. zu einer Nutzung anderweitiger Gebäude. Kay Wendel hielt in seiner Studie zur Unterbringung von Geflüchteten in Deutschland schon im Sommer 2014 fest:

„Die realen Unterbringungsprobleme dieser Monate haben eine lange Vorgeschichte. Über viele Jahre hinweg wurden Unterkunftskapazitäten Zug um Zug abgebaut. Planungen für den Fall einer Zunahme der Asylantragstellerzablen unterblieben weitgehend. Dies wirkte sich in Zeiten zunächst moderat steigender Flüchtlingszahlen nur wenig aus, wird aber nun, während die Ausläufer der größten weltweiten Flüchtlingskrise seit Jahrzehnten auch Deutschland erreichen, zum akuten, kaum noch schnell zu lösenden Problem" (Wendel/ Pro Asyl 2014: 6).

Die neu angekommenen Geflüchteten wurden zum Teil in alten Kasernen, leerstehenden Gewerbeimmobilien, Turnhallen, Zelten oder Containern untergebracht. Die Unterbringungssituation blieb auch bis 2017 angespannt (Hinger/Schäfer 2017). Zu Beginn unseres Projektvorhabens im Frühjahr 2017 fragten wir uns, welchen Einfluss diese angespannte Unterbringungssituation speziell auf queere ${ }^{2}$ Geflüchtete in Deutschland hat. Dabei interessierten uns insbesondere folgende Aspekte:

- Wie gestalteten sich die Wohn- bzw. Unterbringungssituationen ${ }^{3}$ von queeren Geflüchteten? Welche speziellen Probleme und Herausforderungen begegnen ihnen dabei als queere Personen?

- Welche Wünsche und Bedürfnisse äußern queere Geflüchtete in Bezug auf ihre Wohnsituationen? Was sind konkrete Verbesserungsmöglichkeiten?

- Welche Institutionen und Initiativen existieren in Berlin zur Betreuung und Beratung von queeren Geflüchteten zum Thema Wohnen? Wie

2 In diesem Artikel wird der übergreifende Begriff „queer“ benutzt, um Personen mit einer Sexualität, einem Geschlecht und/oder einer Geschlechtsidentität, die von der heterosexuellen und/oder cisgeschlechtlichen Norm abweichen, zu bezeichnen. Wir haben uns gegen die Verwendung der ebenfalls verbreiteten Begriffe LGBTQ* oder LGBTTIQ* entschieden, da wir durch unsere Studie nicht die Erfahrungen aller in diesen Abkürzungen genannten Gruppen abbilden konnten. So haben wir beispielsweise keine Personen interviewt, die sich als bi-sexuell oder inter*geschlechtlich definieren.

3 Wir verwenden in dieser Studie in der Regel den Begriff der „Unterbringung“, da viele Geflüchtete ihren Wohnort nicht selbst wählen können und insbesondere die oft schlechten Bedingungen in Sammelunterkünften unserer Ansicht nach eher eine Form der „Unterbringung“ als des „Wohnens“ darstellen. 
sehen die spezifischen Angebote bzw. Strategien dieser Akteur*innen aus?

Allerdings lagen zu diesem Zeitpunkt kaum Forschungsergebnisse zu diesem Themenkomplex vor. 2017 existierte zwar bereits ein beachtlicher Forschungskorpus zur Unterbringung von Geflüchteten in Deutschland im Allgemeinen (u.a. Christ, Meininghaus und Röing 2017; Deutsches Institut für Menschenrechte 2017; Pieper 2013; Täubig 2009) und im Speziellen zur Unterbringung spezifischer Gruppen Geflüchteter - bspw. Frauen* (Dilger, Dohrn und International Women Space 2016; Foroutan, Hamann, El-Kayed und Jorek/ BIM 2017a). Jedoch ließen sich Anfang 2017 keinerlei Publikationen über die spezifische Situation von queeren Geflüchteten in Bezug auf ihre Unterbringung finden, die die Innenperspektive der Geflüchteten berücksichtigen. Allein LesMigraS (ursprünglich Abkürzung für „lesbische und bisexuelle Migrant_innen, Schwarze Lesben und Trans*"“) - der Antigewalt- und Antidiskriminierungs-Bereich der Lesbenberatung Berlin e.V. - hat 2015 zur Situation von queeren Geflüchteten in Berliner Erstaufnahmeeinrichtungen publiziert (LesMigraS 2015a), fragte dabei jedoch nur die Erfahrungen der Leitungen der Unterkünfte ab. Die Erforschung der Unterbringungsbedingungen für queere Geflüchtete stellte 2017 international wie für Deutschland ein Forschungsdesiderat dar (vgl. Bauer 2017: 22). 2019 erschien mit dem Sammelband „Refugees und Queers: Forschung und Bildung an der Schnittstelle von LSBTTIQ, Fluchtmigration und Emanzipationspolitiken" (Küppers/Bundesstiftung Magnus Hirschfeld) zum ersten Mal in der deutschsprachigen Forschungslandschaft ein Werk, das Forschungen und Aktivismus zur Überschneidung von queeren Lebensweisen und Fluchterfahrungen dokumentiert und bündelt. Carolin Küppers und Kristina Henns konstatierten darin, dass umfassendere wissenschaftliche Erhebungen zu den Bedarfen von queeren Geflüchteten in den Bereichen Asylverfahren, Unterbringung und gesellschaftliche Teilhabe erst im Entstehen sind (Küppers/ Hens 2019: 9).

Diese Forschungslücke in Bezug auf die Lebensverhältnisse queerer Geflüchteter im Allgemeinen bzw. die Wohn- und Unterbringungsverhältnisse im Speziellen motivierte uns zur Durchführung unserer explorativen Kurzstudie. Die Beschäftigung mit den Lebensverhältnissen von queeren Geflüchteten als einer besonders vulnerablen Gruppe birgt jedoch auch immer die Gefahr durch diesen Fokus die Lebensbedingungen der restlichen Geflüchteten aus den Augen zu verlieren, ggf. sogar zu legitimieren und verschiedene Gruppen von Geflüchteten gegeneinander aus- 
zuspielen. ${ }^{4}$ Betrachtungen über die Lebensbedingungen von queeren Geflüchteten sollten daher immer auch im Kontext der Gesamtbehandlung von Geflüchteten gesehen werden. Denn viele der Probleme, die queere Geflüchtete besonders betreffen, speisen sich zu einem großen Teil aus den allgemein schlechten Lebensbedingungen aller Geflüchteter, insbesondere in Sammelunterkünften. Nichtsdestotrotz sind queere Geflüchtete aufgrund ihres Status als Geflüchtete und ihren queeren Lebensweisen von spezifischen Problemlagen in Bezug auf ihre Unterbringung betroffen, die bisher kaum untersucht worden sind, deren Kenntnis jedoch zur Verbesserung der Wohnsituationen queerer Geflüchteter zentral ist.

In der zweiten Jahreshälfte 2017 begannen wir mit unserer qualitativ und explorativ angelegten Kurzstudie zu den Wohnunterbringungen von queeren Geflüchteten mit dem Schwerpunkt Berlin. Wir befragten in qualitativen Einzel- und Gruppeninterviews zwölf queere Geflüchtete zu ihren Erfahrungen sowie zu ihren Bedürfnissen und Wünschen in Bezug auf Wohnen. ${ }^{5}$ Zusätzlich führten wir Interviews mit drei Organisationen, die sich speziell mit den Lebenssituationen von queeren Geflüchteten in Berlin befassen: GLADT (ursprünglich Abkürzung für „Gays \& Lesbians

4 Bilder von muslimischen, männlichen und heterosexuellen Geflüchteten, die „vermeintlich veraltete Werte mit nach Deutschland bringe[n]“ (Küppers/ Hens 2019: 13) und von denen „potentiell Gefahr ausgehe“ (ebd.), die im deutschen medialen Diskurs hergestellt werden, stehen im Gegensatz zu den medialen Bildern über besonders „schutzbedürftige“ queere Geflüchtete, die von ersteren bedroht würden. Es geht hier nicht darum zu negieren, das gewaltvollen Übergriffe gegen queere Geflüchtete durch andere nicht-queere Geflüchtete stattfinden, sondern darum, die zum Teil problematischen, stereotypen und islamfeindlichen Bilder, die in medialen Diskursen in diesem Kontext aufgerufen werden, kritisch zu reflektieren. Des Weiteren muss vermieden werden, ein Bild von Deutschland zu zeichnen, in dem queere Personen vermeintlich keine Diskriminierung mehr durch Deutsche erfahren würden (vgl. Shevtsova 2019: 83-99).

$5 \mathrm{Zu}$ Forschungsethik und Forschungspraxis in der Forschung mit geflüchteten Queers siehe die Beiträge von Zülfukar Çetin, Leonie Teigler und Nina Held im Sammelband „Refugees and Queers“ (Küppers/Bundesstiftung Magnus Hirschfeld 2019). 
aus der Türkei“ “6 ${ }^{6}$, LesMigraS ${ }^{7}$ und der Schwulenberatung Berlin ${ }^{8}$. Queer verstehen wir in diesem Kontext als eine von der heterosexuellen und/oder cisgeschlechtlichen ${ }^{9}$ Norm abweichende Form der Sexualität und/oder des Geschlechts bzw. der Geschlechtsidentität.

Unter den befragten Geflüchteten waren sechs schwule Cis-Männer, eine nicht-binäre ${ }^{10}$ Person mit einer als queer beschriebenen Sexualität, ein transsexueller ${ }^{11}$ und heterosexueller Mann, ein sich hauptsächlich, aber

6 Die Organisation GLADT ist „eine Selbstorganisation von Schwarzen und of Color Lesben, Schwulen, Bisexuellen, Trans*, Inter* und Queere Menschen in Berlin“ (GLADT 2021). Sie engagiert sich „auf unterschiedlichen Ebenen gegen Rassismus, Sexismus, Trans*- und Homofeindlichkeit, Behindertenfeindlichkeit sowie andere Formen von Diskriminierung“ (ebd.). Ein besonderer Schwerpunkt der Arbeit von GLADT „liegt auf den Themen Mehrfachdiskriminierung und Intersektionalität, d.h. den Überschneidungen/Überlappungen und Wechselwirkungen unterschiedlicher Diskriminierungsformen und den damit ergehenden spezifischen Erfahrungen“ (ebd.).

7 LesMigraS ist der Antidiskriminierungs- und Antigewaltbereich der Lesbenberatung Berlin e.V. LesMigraS tritt "gegen alle Formen von Gewalt und Diskriminierung von lesbischen und bisexuellen Frauen, Trans* und Inter* (LBTI)“ ein und setzt sich für eine Gesellschaft ein, „in der alle Aspekte des Lebens und der Persönlichkeit von LSBTI wahrgenommen, akzeptiert und geschätzt werden“ (LesMigraS 2015b).

8 Die Schwulenberatung Berlin bietet Unterstützung bei Fragen, „die das Leben als schwuler oder bisexueller Mann, lesbische Frau, trans- oder intergeschlechtlicher Mensch mit sich bringt.“ (Schwulenberatung 2020a) d.h. „u.a. bei Fragen zur Gesundheit, Drogen und Alkohol, Alter, Identität und Coming Out, Sozialrecht oder Anti-Diskriminierung“ (ebd.). Die Schwulenberatung beherbergt darüber hinaus die Fachstelle für LSBTI* Geflüchtete in Berlin (Schwulenberatung 2020b).

9 Bei Cis-Personen entspricht die Geschlechtsidentität dem Geschlecht, das bei der Geburt in die Geburtsurkunde eingetragen wurde (vgl. Kleiner/ Scheunemann 2016; Dissens e.V. 2020). Personen, die sich als trans* definieren, sind Menschen, deren Geschlechtsidentität nicht dem Geschlecht entspricht, das bei Geburt in ihre Geburtsurkunde eingetragen wurde. „Das Sternchen hinter trans* steht für viele unterschiedliche Selbstbezeichnungen“ (Dissens 2020).

10 „Wenn Menschen sich in den Kategorien «Frau» oder «Mann» nicht repräsentiert sehen, wird das auch non-binäres Geschlecht oder genderqueer genannt [...]. Non-binäres Geschlecht (oder auch nicht-binär) ist [...] ein Oberbegriff, der [...] bedeutet, dass jemand sich nicht in das herkömmliche, streng zweigeteilte Geschlechtersystem einordnen kann oder will" (nonbinary.ch 2021, Hervorhebungen im Original).

11 Der Begriff transsexuell wird von vielen Trans*personen als pathologisierend empfunden, stellt hier jedoch eine explizite Selbstbeschreibung unseres Interviewpartners dar, die er besonders hervorhob. Aus diesem Grund haben wir sie, gemäß seinem Selbstverständnis, wortwörtlich im Text übernommen. Weiter verbreitet ist die Eigenbezeichnung trans* bzw. Trans*mann (siehe Fußnote 9). 
nicht ausschließlich schwul verstehender „Mann mit weiblichem Gefühl“ (Interview mit Navid) sowie drei lesbische Cis-Frauen. Keine der befragten Personen definierte sich im Interview als inter"geschlechtlich ${ }^{12}$, wir gehen daher davon aus, dass alle von uns interviewten Personen dyadisch bzw. endogeschlechtlich ${ }^{13}$ sind. Alle unserer geflüchteten Gesprächspartner*innen waren zwischen 2014 und 2016 aus Syrien (sieben Personen), Russland (drei Personen) oder dem Iran (zwei Personen) nach Deutschland geflüchtet. Die überwiegende Mehrheit unserer Gesprächspartner*innen hatte einen Aufenthaltstitel von drei Jahren als Geflüchtete erhalten, zwei von ihnen (aus Syrien) jedoch nur subsidiären Schutz zwischen einem und dreieinhalb Jahren. Der Asylantrag einer Person aus dem Iran war abgelehnt worden und die Person lebte zum Zeitpunkt des Interviews mit einer kurzfristigen Duldung in Deutschland. Alle Befragten waren in ihrem dritten bis vierten Lebensjahrzehnt und somit recht jung: Mit sieben Personen war die Mehrheit der Befragten Anfang zwanzig (20-24 Jahre), drei waren Mitte bis Ende zwanzig (26-28 Jahre) und zwei Anfang dreißig (30-31 Jahre). Eine der befragten Personen war verheiratet und hatte ein Kind. Der Rest der Personen war unverheiratet, wovon eine Person in einer eingetragenen Lebenspartnerschaft lebte, und kinderlos. Mehrere der Befragten lebten in einer festen Partner*innenschaft und planten zum Teil auch zu heiraten. Die Bandbreite der Berufs- und Schulabschlüsse war sehr groß. Bis auf eine Person verfügten alle Befragten über einen Schulabschluss, sechs von ihnen hatten ein Studium oder eine Berufsausbildung in ihrem Heimatland angefangen, jedoch wegen Flucht oder trans*- und queer"feindlicher Diskriminierung abbrechen müssen. Drei Personen verfügten über eine abgeschlossene Berufsausbildung bzw. ein Studium. Die überwiegende Mehrheit der Befragten identifizierte sich nicht mit einer Ethnizität oder Religion. Zehn der zwölf von uns befragten Geflüchteten lebten zum Zeitpunkt des Interviews in Berlin, zwei Personen in Brandenburg und mit einer Ausnahme hatten alle Befragten ihren Lebensmittelpunkt in Berlin. Viele der Geflüchteten waren jedoch zunächst aufgrund der bestehenden rechtlichen Regulierungen in ein anderes Bundesland

12 Inter*geschlechtliche Personen „sind Menschen, deren körperliches Geschlecht (beispielsweise die Genitalien oder die Chromosomen) nicht der medizinischen Norm von 'eindeutig' männlichen oder weiblichen Körpern zugeordnet werden kann, sondern sich in einem Spektrum dazwischen bewegt" (Queer Lexikon 2021).

13 „Als dyadisch oder endogeschlechtlich werden Menschen bezeichnet, die nicht inter sind, also deren Körper in eine eindeutige medizinische Norm von männlichen bzw. weiblichen Körpern passen" (Queer Lexikon 2020b). 
verteilt worden und hatten seit ihrem Eintreffen in Deutschland an verschiedenen Orten in verschiedenen Formen der Sammelunterbringung gelebt, darunter Turnhallen, Zelte, Container, Gewerbeimmobilien sowie für diesen Zweck angemietete leerstehende Hostels oder Wohngebäude. $\mathrm{Da}$ es uns wichtig war, die Unterbringungssituationen ab dem Eintreffen in Deutschland zu untersuchen, bilden die Untersuchungsergebnisse demnach nicht, wie zunächst beabsichtigt, nur die Wohnunterbringungen in Berlin, sondern auch in anderen Bundesländern ab. Im Erhebungszeitraum wohnten drei der von uns Interviewten noch in einer Sammelunterkunft, davon alle in der queeren Sammelunterbringung der Schwulenberatung. Zwei der Befragten hatten in der Sammelunterbringung der Schwulenberatung gewohnt, waren jedoch zum Zeitpunkt des Interviews schon in Wohngemeinschaften (WGs) umgezogen. Insgesamt wohnten sieben der Befragten in WGs, eine Person mit dem*der Partner*in und eine Person war tage- bis wochenweise in wechselnden Wohnungen oder Hostels untergebracht.

Im Folgenden sollen die Ergebnisse unserer explorativ angelegten Kurzstudie zusammengefasst werden. Dabei betrachten wir die Unterbringungsbedingungen queerer Geflüchteter in allgemeinen Sammelunterkünften (Punkt 2) sowie der speziellen Sammelunterkunft für queere Geflüchtete der Schwulenberatung Berlin (Punkt 3). Darauf aufbauend widmen wir uns den Umgangsstrategien queerer Geflüchteter mit ihren Wohnbedingungen in Sammelunterkünften (Punkt 4) und möchten somit einen Fokus auf die Agency queerer Geflüchteter legen. Im darauffolgenden Abschnitt steht das für die Interviewten zentrale Thema der Wohnungssuche für queere Geflüchtete im Fokus (Punkt 5). Danach erläutern wir die Wohnbedingungen queerer Geflüchteter in privaten Wohnverhältnissen, wie beispielsweise WGs (Punkt 6). Im abschließenden Abschnitt dieses Artikels werden wir ein Fazit unserer Kurzstudie ziehen und einige Empfehlungen zur Wohnunterbringung für queere Geflüchtete geben (Punkt 7).

\section{Unterbringungssituationen queerer Geflüchteter in Sammelunterkünften}

"[T]he system of everything in your life, it's gonna change. The way you sleep, the way you eat, [...] you just have to be there [...]. I couldn't do anything, I just had patience to stay there." (Interview mit Nabil)

Geflüchtete waren bis zum 23.10.2015 nach $₫ 47$ Abs. 1 Asylgesetz (AsylG) verpflichtet bis zu sechs Wochen, längstens jedoch bis zu drei Monaten, in 
der für ihre Aufnahme zuständigen Aufnahmeeinrichtung zu wohnen (Deery/ Republikanischer Anwältinnen- und Anwälteverein 2019: 1). In der Folgezeit wurde die Pflicht zum Wohnen in einer Aufnahmeeinrichtung zunächst auf bis zu sechs Monate und dann erneut durch das sogenannte „Geordnete-Rückkehr-Gesetz“ zum 21.08.2019 auf bis zu 18 Monate ausgedehnt (ebd.). In unserem Untersuchungszeitraum betrug die maximale Wohnverpflichtung in einer Aufnahmeeinrichtung also sechs Monate. Der Wortlaut des $₫ 47$ Abs. 1 S. 1 AsylG gibt dabei eine maximale zeitliche Wohnverpflichtung an. Die Länder konnten während unseres Untersuchungszeitraums also eine Wohnverpflichtung von bis zu sechs Monaten umsetzen, mussten dies jedoch nicht (ebd.: 2).

Gemäß $₫ 3$ Abs. 1 Asylbewerberleistungsgesetz (AsylbLG) gilt während der Unterbringung in einer Erstaufnahmeeinrichtung (EAE) für zu erbringende Sozialleistungen zwingend das Sachleistungsprinzip mit Vollverpflegung, Kleidungsgutscheinen und Taschengeld. Das heißt, Geflüchtete bekommen in dieser Zeit nur ein minimales Taschengeld und haben keine eigenen Kochmöglichkeiten. Darüber hinaus gilt gemäß $₫ 61$ Abs. 1 AsylG für die Dauer der Unterbringung in einer EAE ein absolutes Arbeitsverbot.

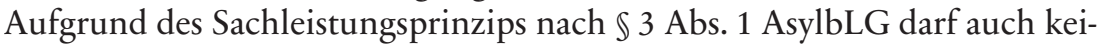
ne Wohnung angemietet werden (Classen/ Flüchtlingsrat Berlin 2017: 121). Nach dem Erhalt des Asylbescheids, längstens jedoch nach einem halben Jahr, werden die Geflüchteten nach einem festen Schlüssel auf die Bundesländer verteilt und sollen „in der Regel in Gemeinschaftsunterkünften untergebracht" werden (gemäß $\$ 53$ Abs. 1 AsylG). In Berlin haben Geflüchtete unmittelbar nach Ende der Verpflichtung in einer EAE zu wohnen, das Recht auf Anmietung einer Wohnung (Wendel/ Antidiskriminierungsberatung Brandenburg 2016: 6).

Geflüchtete werden also nach Stellung ihres Asylantrages zunächst in einer EAE in Form einer Sammelunterkunft untergebracht. Auch nach dem Erhalt des Asylbescheids und Ablauf der Wohnverpflichtung in einer EAE werden sie in der Regel weiterhin in Sammelunterkünften untergebracht, obwohl sie in dieser Zeit zumindest in Berlin theoretisch schon eine Wohnung mieten könnten. Unter Sammelunterkünften verstehen wir sowohl EAEs als auch darauffolgende Unterbringungsformen, in denen viele Personen auf engem Raum untergebracht sind.

Ausnahmen von der erwähnten Wohnverpflichtung in einer EAE können für besonders „schutzbedürftige“ Geflüchtete gemacht werden. Als „besonderes schutzbedürftig“ gelten nach Art. 21 der Richtlinie 2013/33/EU der EU (auch Aufnahmerichtlinie genannt) in nicht abschließender Aufzählung: Minderjährige, Behinderte, ältere Menschen, Schwangere, Alleinerziehende mit minderjährigen Kindern, Opfer von Menschen- 
handel, Personen mit schweren körperlichen Erkrankungen, Personen mit psychischen Störungen, Personen, die Folter, Vergewaltigung oder sonstige schwere Formen psychischer, physischer oder sexueller Gewalt erlitten haben (Wendel/ Antidiskriminierungsberatung Brandenburg 2016: 4; Europäisches Parlament/ Rat der Europäischen Union 2013: 106). Queere Geflüchtete sind in dieser Aufzählung zwar nicht explizit benannt, könnten nach dem Ermessen des zuständigen EU-Mitgliedsstaates jedoch auch unter diese Richtlinie fallen (Flüchtlingsrat Baden-Württemberg 2020)14. In Berlin werden queere ${ }^{15}$ Geflüchtete in der Tat seit August 2015 im erweiterten Sinne als besonders „schutzbedürftig“ definiert:

\section{„Zumindest im erweiterten Sinne sind auch von Gewalt bedrohte Frauen sowie Lesben, Schwule, Bisexuelle und trans- und intergeschlechtliche Men- schen (LSBTI), die als Flüchtlinge in Berlin um Asyl nachsuchen, als beson- ders schutzbedürftige Personengruppen zu verstehen." (Senat von Berlin 2015: 12)}

Zum Forschungszeitpunkt wurden jedoch laut Aussagen unserer Interviewpartner*innen queere Geflüchtete in Berlin faktisch nicht von der Wohnverpflichtung in einer EAE entbunden (Interview mit Mahmoud Hassino, Schwulenberatung). Viele unserer Gesprächspartner*innen wohnten darüber hinaus auch noch anderthalb Jahre nach ihrem Ankommen und nach dem Erhalt ihres Asylbescheids in einer Sammelunterkunft, da sie keine bezahlbare Wohnung oder WG finden konnten. Die Sammelunterkünfte, die zunächst als temporäre Lösung zur Unterbringung gedacht waren, sind dadurch oft zu einem dauerhaften Wohnort für viele (queere) Geflüchtete geworden. Deshalb sollen im Folgenden die Wohnbedingungen für queere Geflüchtete in Sammelunterkünften beleuchtet werden. Dabei haben wir uns auf solche Faktoren fokussiert, die spezielle Auswirkungen auf queere Geflüchtete aufgrund ihrer queeren Lebensweisen haben. Durch die Interviews mit unseren Gesprächspartner*innen kristallisierten sich die Aspekte Auslastung und Privatsphäre, Sicherheit und sozialarbeiterische Betreuung in sowie Lage von Unterkünften und die psychische Gesundheit der Befragten heraus.

142019 gab der Ausschuss für Inneres und Heimat des Deutschen Bundesrates eine Beschlussempfehlung heraus, nach der auch lesbische, schwule, bi-, trans- oder intersexuelle Personen nunmehr als schutzbedürftig gelten (Deutscher Bundesrat 2019: $14 \mathrm{f}$.).

15 Der Berliner Senat benutzt den Begriff „LSBTI“. 


\subsection{Auslastung und Privatsphäre}

"I couldn't stay there at all [...], hundred beds near together, and all the people, I couldn't even change my clothes. When I go to the bathroom, I should be naked there, and for me it's not about being naked, it's about the homophobic [people], they are looking at me being naked, and they are saying: oh, what is that! What kind of body do you have! [...] they wanna joke. So it was not easy at all." (Interview mit Jamal)

Je größer die Unterkünfte waren, desto stressiger wurde das Leben darin durch unsere Interviewpartner*innen empfunden. Insbesondere der Mangel an Privatsphäre war eklatant und einer der Hauptgründe, weshalb sich Geflüchtete unwohl in Sammelunterkünften fühlten. Die Unterkünfte waren zum Teil überbelegt und so mussten sich bis $\mathrm{zu}$ acht Personen einen durch Raumteiler abgeteilten Bereich teilen. Manche Unterkünfte wurden im Laufe des Aufenthalts unser Gesprächspartner*innen noch aufgestockt oder durch temporäre Anbauten wie Zelte oder Container ergänzt, um mehr Menschen dort unterzubringen, was die Unterbringungssituation weiter verschlechterte. Zelte, Turnhallen oder Gebäude mit Raumteilern wurden als besonders schlechte Unterkünfte erlebt, da hier durch fehlende Zimmer am wenigsten Privatsphäre gegeben war. Zudem werden in solchen Unterkünften die Lichtanlagen zentral gesteuert und somit gibt es feste Zeiten, zu denen das Licht an- bzw. ausgeschaltet ist. Darüber hinaus sind in solchen Unterkunftstypen die vorhandenen Sanitäreinrichtungen oft nicht auf eine große Anzahl an Benutzer*innen ausgerichtet. Interviewpartner"innen berichteten, dass zum Teil 50 Personen oder mehr dasselbe Bad benutzen mussten.

Diese Bedingungen wirken sich in besonderer Weise auf queere Geflüchtete aus. Jamal, von dem das einleitende Zitat dieses Abschnitts stammt, war zunächst in einer Turnhalle untergebracht und konnte nach zehn Tagen die Verlegung in eine andere Unterkunft mit der Begründung erwirken, dass er „sichtbarerweise“ schwul sei und daher in der Turnhalle aufgrund der fehlenden Privatsphäre Diskriminierung ausgesetzt sei. Insbesondere die gemeinsame Benutzung von sanitären Anlagen durch viele Personen ist für queere Geflüchtete oft sehr problematisch, wenn sie durch ihr Aussehen und/oder Verhalten nicht als heterosexuell und/ oder cis passen ${ }^{16}$ können. Das gilt insbesondere für Trans*personen, die

16 Passen, abgeleitet vom englischen Verb to pass (zu Deutsch in etwa als etwas oder jemand durchgehen) bedeutet im Kontext von Trans*geschlechtlichkeit, dass einer Person die Geschlechtsidentität von außen zuerkannt wird, der sich 
in unterschiedlichen Stadien der Transition gezwungen sind mit Cis-Personen gemeinsame Duschen zu benutzen und dabei oft Diskriminierung erleben (Interview mit Mahmoud Hassino, Schwulenberatung). Darüber hinaus sei es aufgrund von Trans*feindlichkeit für Trans*personen zum Teil schwer, andere Gemeinschaftsräume, wie Mensen, zu nutzen. In der Regel haben Geflüchtete aufgrund der erwähnten rechtlichen Regulierungen in den ersten sechs Monaten ihrer Unterbringung, zum Teil jedoch auch deutlich länger, keinen Zugang zu Kochmöglichkeiten und müssen das Verpflegungsangebot aus den zentralen Kantinen verzehren. Dies kann zur Isolation von Trans*personen führen. So wurde uns von einem Fall berichtet, in dem Sozialarbeiter*innen einer von Diskriminierung betroffenen Trans"person empfahlen, nicht mehr in der gemeinsamen Cafeteria zu essen (Interview mit Masha, LesMigraS). Insgesamt sähen sich Trans*personen in Sammelunterkünften mit einer besonders schwierigen Lage konfrontiert, denn Transfeindlichkeit, insbesondere Trans* misogynie ${ }^{17}$, seien weit verbreitet (Interview mit Mahmoud Hassino, Schwulenberatung).

Zusätzlich zur Überbelegung und dem Mangel an Privatsphäre in den Unterkünften fehlen oft auch sinnvolle Beschäftigungsmöglichkeiten für die Geflüchteten. Denn sie dürfen in der Regel aufgrund der erwähnten rechtlichen Regulierungen zumindest während des Aufenthalts in der EAE nicht arbeiten, warten oft monatelang auf die Bewilligung eines Deutschkurses und besitzen im Allgemeinen kaum finanzielle Mittel, um Hobbies oder Beschäftigungen außerhalb der Unterkunft nachgehen zu können. Die Überschneidung dieser Umstände führt immer wieder zu Spannungen unter den Bewohner*innen (vgl. Christ et al. 2017). So wurde in den Interviews zum Teil von aggressivem oder gewalttätigem Verhalten oder von erhöhtem Drogen- oder Alkoholkonsum einzelner Bewohner*innen berichtet. Unser*e Interviewpartner*in von LesMigraS hob bei der Betrachtung solcher Spannungen die Notwendigkeit der Kontextualisierung hervor:

"I think it is also a very racist technology not to speak about the problem. And the problem is that people are not having good living conditions and

die Person auch selbst zugehörig fühlt (vgl. Queer Lexikon 2020a), kann aber auch verwendet werden, um queere Personen zu beschreiben, die von anderen Personen als heterosexuell eingeordnet werden (heterosexuelles Passing).

17 Trans*misogynie ist ein Begriff, der die Diskriminierung von Trans*frauen beschreibt. Dabei geht es darum, die Überkreuzung von Trans*feindlichkeit und Frauenfeindlichkeit (Misogynie) zu benennen und aufzuzeigen, dass Trans*frauen durch Trans*misogynie eine spezifische Form der Diskriminierung erfahren (vgl. Queer Lexikon 2020c). 
are living for a long time together, sharing rooms with strangers and maybe also with people from their own background or maybe from different backgrounds. It's not nice for everyone. And it is not about nationality or language or our background [...] if you would put so many white Germans in one room, they would also fight each other." (Interview mit Masha, LesMigraS).

Die von uns befragten Organisationen waren sich daher darin einig, dass Sammelunterbringung generell die falsche Form der Wohnunterbringung für queere Geflüchtete bzw. für Geflüchtete im Allgemeinen ist.

\subsection{Sicherheit}

"Safety, ja, they [queer refugees] very often tell us in our counseling about discrimination, about some traumatic experience or about how not having a safe place doesn't give them the possibility to reflect about all the bad things that happened in the past. Or even direct violence or discrimination happens in these shelters. And I have heard so many sad stories." (Interview mit Masha, LesMigraS)

Viele der von uns befragten Geflüchteten fühlten sich in Sammelunterkünften nicht sicher. Waren sie geoutet bzw. passten nicht als heterosexuell oder cis, erlebten sie oft Diskriminierung durch andere Mitbewohner*innen. Einige Geflüchtete berichteten auch von Problemen mit dem Sicherheitspersonal der Unterkünfte, das sich ihnen gegenüber diskriminierend äußerte. Zum Teil standen die Unterkunftsleitungen diesen Vorgängen tatenlos gegenüber. Waren unsere Gesprächspartner*innen nicht „out", lebten sie in der Unterkunft oft in der Angst geoutet zu werden. Einige der von uns Interviewten berichteten davon, über lange Zeit in den Unterkünften vorgegeben zu haben, dass sie heterosexuell seien. Das ging oft mit einem Wandel des eigenen Verhaltens und der Kleidung einher und führte zu dem Gefühl, nicht „man selbst“ zu sein. Dies beschrieben diese Interviewpartner*innen als sehr belastende und entfremdende Situation. Der Interviewpartner Navid erzählte in diesem Kontext, dass sich seine Zeit in der Sammelunterkunft angefühlt habe, wie in einem Gefängnis zu sein, obwohl er sich von seiner Flucht nach Deutschland erhofft hatte, seine Sexualität und Geschlechtsidentität endlich frei ausleben zu können. Ein weiterer Interviewpartner namens Lee wurde, nachdem er sich vor einem Mitbewohner geoutet hatte, per Facebook bedroht. Nach seinem Auszug aus der Unterkunft wurde ihm damit gedroht, ihn vor seiner gesamten Familie zu outen. Selbst nachdem er die Unterkunft ver- 
lassen hatte, war er somit nicht vor Diskriminierung durch ehemalige Mitbewohner*innen gefeit. Andere Gesprächspartner*innen isolierten sich aus Angst vor homofeindlichen Übergriffen, was zu Vereinsamung innerhalb der Unterkunft führte. In vielen Unterkünften werden Wohngruppen darüber hinaus nach Ländern oder Sprachen gebildet. Das führt jedoch zum Teil dazu, dass queere Geflüchtete auch mit konservativ eingestellten Landsleuten zusammenleben müssen. Masha von LesMigraS unterstrich daher, dass Sicherheit für queere Geflüchtete von besonderer Wichtigkeit sei, was insbesondere bedeute, ein eigenes Zimmer in der Unterkunft zu haben.

Ein anderer Gesprächspartner namens Jamie, der mehrere Monate in einer sehr großen Unterkunft gelebt hatte, fühlte sich vor allem durch externe rechte Angriffe bedroht. Die Unterkunft wurde regelmäßig von Neonazis angegriffen und es wurde versucht, sie mit Molotov-Cocktails in Brand zu setzen. Die Unterkunft verfügte daher auch über Polizeischutz und die Einlasskontrollen waren sehr streng. Er hatte Angst das Gebäude $\mathrm{zu}$ verlassen, insbesondere zur Abendzeit, was dazu führte, dass er die meiste Zeit in der Unterkunft verbrachte. Er verließ sie jedoch trotzdem manchmal, um der Enge und der ständigen Präsenz seiner Zimmergenoss*innen zu entfliehen, obwohl er dies als gefährlich empfand.

\subsection{Sozialarbeiterische Betreuung}

„And then these persons [Mitarbeiter"innen der Unterkunft] are maybe not so sensitized for LGBT-topics. And there is no trust, and it is maybe not so transparent what would happen if I contacted somebody who is a social worker in the Unterkunft. Would this person say it to the job-center? Would this person say it to the BAMF? How does this system work? I think there is lots of mistrust." (Interview mit Masha, LesMigraS)

Eine weitere Problematik war in vielen Unterkünften, dass es an Bewusstsein für die Lebensrealitäten von queeren Geflüchteten fehlte. Mitarbeiter*innen der Unterkünfte sind meist nicht sensibilisiert für eine Unterstützung queerer Geflüchteter. ${ }^{18}$ Gleichzeitig fehlte es vielen queeren Geflüchteten an Vertrauen, um mit Sozialarbeiter*innen der Unterkünfte über ihre Probleme zu sprechen, auch weil sie Angst hatten, dass

18 Vertiefend zu dieser Thematik ist der Beitrag „queer und hier - Sensibilisierung von Sozialarbeitenden in Unterkünften“ (2019) von Gabriele Fischer, Nadine Ober und der Projektgruppe der Hochschule Esslingen. 
sie anschließend gegenüber Dritten geoutet werden. LesMigraS und die Schwulenberatung bieten daher gemeinsam Sensibilisierungsworkshops für Personal an, das in Geflüchtetenunterkünften arbeitet. Dabei sollen Sozialarbeiter*innen, die nicht unbedingt in queeren Kontexten arbeiten, für queere Lebensweisen und Bedürfnisse sensibilisiert werden, um ihre queeren Klient"innen besser beraten und unterstützen zu können. ${ }^{19}$

Insgesamt wurde die Betreuung durch Sozialarbeiter*innen sehr unterschiedlich erlebt. Manche Interviewpartner*innen berichteten von sehr engagierten und bemühten Sozialarbeiter"innen, die sie in vielen Anliegen unterstützt und sich auch bemüht hätten, auf die speziellen Situationen queerer Geflüchteter einzugehen. So boten beispielsweise einige Sozialarbeiterin*innen ihren queeren Klient*innen eine Verlegung in eine kleinere Unterkunft oder ein Einzelzimmer an. Andere Geflüchtete schilderten hingegen überwiegend schlechte Erfahrungen mit Sozialarbeiter"innen, von denen sie sich nicht oder kaum unterstützt fühlten. So wurde beispielsweise berichtet, dass die Sozialarbeiter*innen aufgrund der Fülle ihrer Klient*innen kaum Zeit für die Einzelnen hatten. Mehrere Gesprächspartner*innen erzählten zudem, dass die Fluktuation unter den Sozialarbeiter"innen der Unterkünfte sehr hoch war und sie oft nur einige Monate dort arbeiteten. Dies wurde von den Geflüchteten als frustrierend erlebt, da sich dadurch jedes Mal ihr*e Ansprechpartner*in änderte. Eine als gut empfundene sozialarbeiterische Begleitung korrelierte mit einer eher hohen Zufriedenheit mit der Unterkunft. Dies deutet darauf hin, dass sie ein sehr wichtiger Faktor in der Qualität der Unterkünfte ist.

\subsection{Lage}

"[T]his isolation, these Unterkünfte are often in some places at the edge of Berlin or, if it is Brandenburg, in some other cities in the middle of nowhere. There is no supermarket, no gym, no swimming pool, no [...] bar or cinema. And it is an isolation from the society. And okay, in Berlin it is a bit different. But there are also places somewhere far in Marzahn or in Blankenfelde where people have to drive one and a half hours to their courses or to their institutions [...]" (Interview mit Masha, LesMigraS)

19 Masha von LesMigraS schlug darüber hinaus einen ähnlichen Sensibilisierungsansatz für Mitarbeiter*innen von Behörden vor, um rassistischen und queer*feindlichen Behandlungen vorzubeugen. 
Ein weiteres Problem in der Sammelunterbringung queerer Geflüchteter ist, dass viele Unterkünfte in städtischen Randgebieten oder auf dem Land angesiedelt sind. Viele unserer geflüchteten Interviewpartner*innen sind nach der Erstregistrierung infolge des bereits beschriebenen Verteilungsmechanismus von Geflüchteten auf die verschiedenen Bundesländer, von einer Großstadt in eine Unterkunft in einem anderen Bundesland verlegt worden. So wurden viele unserer Gesprächspartner*innen zwei- bis dreimal verlegt, bevor sie in ihrer endgültigen Unterkunft ankamen. Einerseits fehlte in peripheren Unterkünften eine allgemeine infrastrukturelle Anbindung (vgl. Sauer und Vey 2019), andererseits gab es in der Umgebung keine queere Community mit queeren Beratungsstellen oder Orten, an denen man andere Queers treffen kann. Für viele queere Geflüchteten ist es jedoch enorm wichtig, in einer Großstadt wie Berlin untergebracht zu werden, weil es dort eine spezielle Unterkunft und eine vergleichsweise große queere Community mit entsprechenden Unterstützungsangeboten gibt. Hassino bemängelte, dass, auch wenn er queeren Geflüchteten aus anderen Bundesländern eine Platzzusage in der Unterkunft der Schwulenberatung anbieten könnte, der Umzug nach Berlin nicht bewilligt werde. Zwar könnte in speziellen Fällen dieser Verteilungsmechanismus ausgesetzt werden, allerdings werde diese Ausnahme bei queeren Geflüchteten nicht gemacht. Mehrere Geflüchtete berichteten hingegen davon, in abgelegenen Kleinstädten oder Dörfern untergebracht worden zu sein, in denen zum Teil nur zweimal am Tag ein Bus zur nächsten Stadt fuhr. In sehr abgelegenen Unterkünften gab es darüber hinaus zum Teil auch kein Internet, was zur physischen Mobilität zusätzlich die digitale Mobilität der Bewohner*innen einschränkte. Diese Situation empfanden alle davon betroffenen Geflüchteten als sehr negativ. Ein weiteres Problem war, dass in diesen kleinen Ortschaften keinerlei anonymes Leben möglich war. Die von uns befragten queeren Geflüchteten hatten daher Angst, in diesem Umfeld geoutet zu werden. Einige Interviewpartner*innen berichteten ebenfalls, dass ihnen viele der Dorfbewohner*innen nicht freundlich gesinnt waren, weil sie queere Personen und/oder of Color sind. Die Isolation in Bezug auf queeres Leben in diesen Umgebungen führte bei einigen Befragten zu Antriebs- und Lustlosigkeit bis hin zur Depression. Ein befragter Geflüchteter forderte, dass es insbesondere für Trans"personen, speziell für Trans"frauen und anderweitig vulnerable Geflüchtete, die Möglichkeit des Umzugs in eine Großstadt geben müsse. Ein anderer Gesprächspartner wünschte sich mehr Informationen darüber, wie feste Partner*innen, die ebenfalls geflüchtet sind und die nicht in Berlin leben, nach Berlin ziehen können. 


\subsection{Psychische Gesundheit}

"I mean the need for personal space and privacy [...] you need it to feel healthier, to be better, to at least reflect on your psychological problems if you are going through therapy and those things." (Interview mit Mahmoud Hassino, Schwulenberatung)

Jenseits der genannten Faktoren, die durch die Unterbringung selbst bestimmt sind, ergab sich, dass auch der psychische Zustand queerer Geflüchteter einen Einfluss auf ihr Wohlbefinden in Sammelunterbringungen hat. Einige unserer Gesprächspartner*innen litten unter einer posttraumatischen Belastungsstörung und/oder einer Depression durch Ereignisse, die sie vor, während oder nach der Flucht erlebt haben. Unsere Interviewpartner*innen berichteten von physischen und sexualisierten Gewalterfahrungen und Misshandlungen, homo und trans"feindlicher Diskriminierung und Verfolgung durch staatliche Akteur*innen wie Polizeibehörden oder nicht-staatliche Akteur*innen wie den Daesh ${ }^{20}$. Daneben hatten viele unserer Gesprächspartner*innen auch den Verlust von Angehörigen, Partner*innen und Freund*innen durch Krieg und/oder Flucht zu beklagen. Diese traumatischen Erfahrungen führten bei einigen der Befragten zu Gedächtnislücken, Schlafstörungen und anderen psychosomatischen Symptomen sowie in drei Fällen zu Suizidgedanken, in zwei Fällen auch zu Suizidversuchen. Mehrere Befragte waren deswegen in psychiatrischer oder psychologischer Behandlung bzw. suchten regelmäßig die psychologischen Beratungsstellen von LesMigraS oder der Schwulenberatung auf. Eine 2020 veröffentliche Studie der Universität Leipzig geht davon aus, dass ca. die Hälfte aller Geflüchteter, die zwischen Mai 2017 und Juni 2018 in Leipzig angekommen sind, Anzeichen einer psychischen Störung zeigten (Nesterko, Jäckle, Friedrich, Holzapfel und Glaesmer 2020). Auch Ergebnisse aus den Niederlanden und Österreich, die allerdings nicht repräsentativ sind, deuten darauf hin, dass queere Geflüchtete in besonderem Maße von posttraumatischen Belastungsstörungen betroffen sind (Alessi, Kahn, Woolner und Van der Horn 2018). Golembe, Leyendecker

20 Daesh ist das latinisierte Akronym der arabischen Bezeichnung الدولــة الإســـامية في العــر اق و الثــام Deutsch übersetzt bedeutet „Islamischer Staat im Irak und der Levante“. Das ebenfalls oft verwendete Akronym „IS“ für „Islamischer Staat“ ist eine wortwörtliche Übersetzung der Eigenbezeichnung الدولة الإسـلامية („Al-Daula al-Islamija“). Die Selbstbezeichnung IS bildet den Anspruch ab, der einzige islamische Staat der Welt zu sein. Mit der Verwendung des Begriffs Daesh statt der Bezeichnung IS soll dieser Anspruch zurückgewiesen werden (vgl. Deutschlandfunk 2015). 
und Busch (2019) stellen zusammenfassend fest, dass nach der Ankunft in ihrem Zielland queere Geflüchtete vermehrt von Depressionen, Angst, traumatischem Stress, gesteigertem Drogenkonsum sowie Suizidgedanken berichten. Die Publikation von umfassenderen Studienergebnissen der Universität Leipzig zu den Erfahrungen mit sexualisierter Gewalt und psychischer Gesundheit von queeren Geflüchteten steht zum Zeitpunkt des Verfassens dieses Artikels noch aus. Aufgrund der erwähnten Studienergebnisse und der von unseren geflüchteten Gesprächspartner*innen berichteten Diskriminierung und Angriffe, die sie auf der Flucht oder nach ihrer Ankunft in Deutschland erlebt haben, ist jedoch davon auszugehen, dass queere Geflüchtete wahrscheinlich in besonderem Maße in ihrer psychischen Gesundheit gefährdet sind. Die Unterbringung in Sammelunterkünften vergrößert die psychologischen Probleme der Geflüchteten oftmals weiter. Insbesondere der Mangel an Privatsphäre in den Sammelunterkünften stellt in diesem Kontext ein Problem dar, wie das Eingangszitat zu diesem Abschnitt aufzeigt. Es fehlen Rückzugsräume, die für betroffene queere Geflüchtete, aber auch andere Geflüchtete mit traumatischen Erfahrungen, dringend notwendig wären.

\section{Queere Sammelunterkunft der Schwulenberatung}

„Und alle [...] die da [Unterkunft für queere Geflüchtete der Schwulenberatung] drin sind, wollen auch so schnell wie möglich raus, weil natürlich, wie jede andere Unterkunft, ist die Unterkunft generell ein Problem [...] also es ist problematisch, Leute in so engem Raum da reinzustecken." (Interview mit GLADT)

Nachdem wir im vorherigen Abschnitt die Unterbringungsbedingungen der von uns befragten queeren Geflüchteten in allgemeinen Sammelunterkünften zusammengefasst haben, möchten wir uns nun den Unterbringungsbedingungen in der speziell auf queere Bewohner*innen ausgerichteten Sammelunterbringung der Schwulenberatung Berlin widmen. Als der Berliner Senat im Spätsommer 2015 „LSBTI-Geflüchtete“ zu einer Gruppe besonders schutzbedürftiger Geflüchteter im erweiterten Sinne erklärt hatte, nutzte die Schwulenberatung Berlin diesen Umstand. Sie beantragte und plante eine spezielle Unterkunft für queere Geflüchtete. Die Schwulenberatung hatte zunächst dezentrale Wohnstätten für queere Geflüchtete betreiben wollen. Beabsichtigt war es, Wohnungen für jeweils ca. sechs bis sieben Personen zu schaffen, wobei einmal am Tag ein"e Sozialarbeiter*in die Wohnung besuchen sollte. Dieses Konzept wurde jedoch vom Senat 
mit der Begründung abgelehnt, dass es nicht mit dem geltenden Gesetz in Berlin in Bezug auf die Wohnverpflichtung in einer Sammelunterkunft zu vereinbaren sei (Interview mit Mahmoud Hassino, Schwulenberatung). Der Senat bewilligte jedoch eine Unterkunft für queere Geflüchtete in Form einer Sammelunterkunft. Infolgedessen betreibt die Schwulenberatung seit 2016 eine eigene Unterkunft für queere Geflüchtete mit ca. 120 Plätzen. Ein Teil der Unterkunft besteht aus Wohnungen, die als EAE fungieren und daher nicht über einen Zugang zu einer Küche verfügen. Der andere Teil der Sammelunterkunft, der als Folgeunterbringung auf die EAE gedacht ist, verfügt über Wohnungen mit Küchenzugang. Auch bei der Unterkunft der Schwulenberatung handelt es sich um eine Form der Sammelunterkunft, die jedoch speziell auf eine queere Zielgruppe ausgerichtet ist. Das bedeutet u.a., dass die Unterkunft ein spezielles Sicherheitskonzept besitzt und Sozialarbeiter*innen beschäftigt, die mit queeren Lebensweisen vertraut oder selbst queer sind. Im Folgenden sollen analog zu den Unterbringungsbedingungen queerer Geflüchteter in konventionellen Sammelunterkünften die Wohnumstände in der queeren Unterkunft der Schwulenberatung vorgestellt werden. ${ }^{21}$

\subsection{Auslastung und Privatsphäre}

„So, this mass housing, even our shelter [...] on paper it's good compared to others in Berlin. But you still have four, five people [...] in one room. This is not acceptable." (Interview mit Mahmoud Hassino, Schwulenberatung)

Unser*e Interviewpartner*in von GLADT bemängelte, dass die Unterkunft seit ihrem Bestehen immer voll belegt war. Die Unterkunft mit 120 Plätzen konnte bei Weitem nicht den Bedarf, der 2017 in Berlin vorhanden war, abdecken. Mehrere von uns interviewte Bewohner*innen oder ehemalige Bewohner*innen beklagten sich über die hohe Auslastung und räumliche Enge in der Unterkunft. Insbesondere für Geflüchtete, die lange in der Unterkunft leben müssen, ist dies problematisch. Die von uns Interviewten lebten zum Teil seit einem Jahr und vier Monaten in der Unterkunft. Unsere geflüchteten Gesprächspartner*innen schilderten uns, dass sie mit bis zu fünf anderen Personen eine kleine Wohnung in der Unterkunft

21 Der Punkt „Lage der Unterkunft“ entfällt hierbei, da die Unterkunft der Schwulenberatung im Innenstadtgebiet Berlins liegt. Probleme bezüglich der Lage der Unterkunft, wie wir sie für peripher gelegene Unterkünfte ausmachen konnten, ergaben sich hier nicht. 
bewohnten. In Arefs Wohnung gab es beispielsweise zwei Zimmer und ein Durchgangs-Wohnzimmer. Er bewohnte das Durchgangszimmer, die anderen zwei Zimmer teilten sich jeweils zwei Personen. Jedoch hatte eines der benachbarten Zimmer keine Tür, sodass die ohnehin schon minimale Privatsphäre im Durchgangszimmer noch stärker eingeschränkt war. Aref fühlte sich durch diese Umstände massiv in seiner Privatsphäre und seinem Tagesrhythmus gestört:

„Das erste Problem ist, dass ich in den Wohnungen nicht sagen kann, ich möchte schlafen und dann auch schlafen kann. Das geht nicht. Weil, das ist eine Gemeinschaftswohnung. Ich schlafe zum Beispiel um zwölf, eine andere Person um zwei Ubr in der Nacht. [...] Das ist verschieden. Weil, sie gehen nicht in die Schule, ich gehe in die Schule. Das ist manchmal das Problem." (Interview mit Aref)

Hinzu kommt, dass Aref sich als trans*sexueller Mann zusammen mit cisgeschlechtlichen Personen die WG teilte. Da er im Durchgangzimmer wohnte, sei beispielsweise die Privatsphäre beim Wechseln der Kleider oft nicht oder nur ungenügend gewährt. Er wünschte sich, dass Trans*personen eigene Zimmer erhalten, was jedoch aufgrund der Auslastung der Unterkunft nicht möglich sei. Er unterstrich, dass auch feminin auftretende schwule Cis-Männer ein ähnliches Problem hätten. Es gebe in der Unterkunft keine Wohnung allein für Trans*personen. Mitarbeiter*innen der von uns interviewten Organisation und andere Bewohner*innen machten hier allerdings gegenteilige Aussagen, so dass sich vermuten lässt, dass es je nach Anzahl der in der Unterkunft wohnenden Trans*personen und der allgemeinen Auslastung manchmal separate Trans"wohnungen gibt, manchmal jedoch auch nicht. Auch Kim, eine cisgeschlechtliche lesbische Bewohnerin der Unterkunft, wünschte sich eine Wohnung, in der sie nur mit Frauen wohnen könnte, wobei sie jedoch stark trans*feindlich argumentierte und Trans*frauen hier ausschloss.

\subsection{Sicherheit}

„Und ich habe wirklich da [in der Unterkunft der Schwulenberatung] viel Stress, weil viele Leute da sind, die mich schlecht angucken, (...) weil, am Anfang habe ich noch keine Hormone bekommen (...) Nach zwei Monaten habe ich eine Hormontherapie angefangen und ab da habe ich viel Stress bekommen (...).“ (Interview mit Aref) 
Es wurden einige Vorkehrungen getroffen, um die Sicherheit der Bewohner*innen der Unterkunft der Schwulenberatung nach außen zu gewährleisten. So wird die Adresse der Unterkunft nicht veröffentlicht. Des Weiteren gibt es Sicherheitspersonal vor Ort; bei Angriffen wird die Polizei verständigt. Trotz dieser Sicherheitsmaßnahmen verwies Masha vom LesMigraS darauf, dass eine queere Unterkunft auch zum Problem für darin lebende Geflüchtete werden könnte, wenn der Ort der Unterkunft bekannt und infolgedessen angegriffen werde. Einen solchen bewaffneten, externen Angriff auf die Unterkunft hatten einige unserer Interviewpartner*innen bereits erlebt.

Alle Bewohner*innen der Unterkunft berichteten daneben von Spannungen zwischen den Bewohner*innen. Einige Bewohner*innen hatten auch von physischen Übergriffen innerhalb der Unterkunft gehört oder diese selbst miterlebt. Insgesamt gab es viel Misstrauen gegenüber anderen Bewohner"innen, ob diese „wirklich" queer seien oder nur in einer besseren Unterkunft untergebracht werden wollten. Hassino verwies ebenfalls darauf, dass es viel bi-feindliches Verhalten unter den Bewohner*innen gebe.

Bewohner*innen der Unterkunft wie auch unser*e Interviewpartner*in von GLADT berichteten uns darüber hinaus, wie im obigen Zitat geschildert, von Trans"feindlichkeit in der Unterkunft. Eine cisgeschlechtliche Bewohner"in der Unterkunft machte während des Interviews beispielsweise mehrmals trans"feindliche Aussagen und äußerte Angst vor und Ablehnung der Trans*person, mit der sie sich eine Wohnung teilte. Trans*personen seien nach Aussagen anderer Interviewter auch häufiger Übergriffen innerhalb der Unterkunft ausgesetzt.

\subsection{Sozialarbeiterische Betreuung}

"I think the problem for me is [that] they [the social workers] are all the time working with the gay refugees and they don't help the lesbian refugees. They like working with the gay guys more. But now we have [...] a female social worker, she helps us. Well, for me it is easier to speak with a female social worker." (Interview mit Kim)

Wie auch in anderen Unterkünften wurde in der Unterkunft der Schwulenberatung die große Fluktuation der Sozialarbeiter*innen von Bewohner*innen als problematisch empfunden. Ein Interviewpartner berichtete, dass die Sozialarbeiter*innen anfangs ca. sechs Monate blieben, jetzt aber ca. jeden Monat neue kämen und besonders die engagierten oft schneller 
wieder gehen würden. Auch hier waren, wie in anderen Einrichtungen, die Erfahrungen mit Sozialarbeiter*innen durchwachsen, manche Sozialarbeiter*innen wurden als engagiert, andere als weniger hilfreich empfunden, jedoch waren nach Aussagen der Geflüchteten alle Sozialarbeiter*innen mit queeren Lebensweisen vertraut oder selbst queer.

Der sich als trans"sexuell positionierende Bewohner Aref und die cisgeschlechtliche lesbische Bewohnerin Kim fühlten sich durch die vorhandenen Sozialarbeiter*innen schlechter betreut als die anderen, überwiegend cisgeschlechtlichen schwulen Bewohner der Unterkunft, wie das Eingangszitat dieses Abschnitts unterstreicht. Aref gab an, dass es in der Unterkunft nur einen Sozialarbeiter gebe, der selbst trans* sei. Für ihn war es jedoch sehr wichtig, mit seinen körperlichen Beschwerden, die sich durch die Transition ergaben, die sozialarbeiterische Unterstützung einer Person zu erhalten, die selbst trans* sei und daher seine Probleme nachvollziehen könne.

Neben professionellen Sozialarbeiter*innen arbeiteten auch ehrenamtliche Freiwillige in der Unterkunft mit. Sie erkundigten sich bei den Geflüchteten, welche Probleme sie hätten oder ob sie etwas bräuchten, brachten Essen vorbei und boten Mentor"innenschaften oder Workshops an. Ein Vorteil, den die queere Unterkunft hier bietet, ist eine enge Verbindung zur und Austausch mit der queeren Community in Berlin. Einige Bewohner*innen der Unterkunft schilderten, dass das Engagement durch Freiwillige zu Beginn ihres Aufenthalts in der Unterkunft (ab Anfang 2016) größer gewesen sei. Dies könnte darauf hindeuten, dass im Verlauf von $2016 / 17$ die Bereitschaft der queeren Community in Berlin, sich für queere Geflüchtete zu engagieren, abgenommen hat.

\subsection{Psychische Gesundheit}

"One time I got really drunk and I tried to cut my wrists and I tried to commit suicide and then the ambulances came, and they saved me" (Interview mit Ahmad)

Wie bereits in Punkt 2.5 geschildert, legen die Gespräche mit unseren Interviewpartner*innen nahe, dass queere Geflüchtete auf ihrer Flucht und/oder danach oft Queer*feindlichkeit oder anderen Formen der Diskriminierung ausgesetzt waren, sich dies negativ auf ihre psychische Gesundheit auswirkt und einige daher mit psychischen Problemen zu kämpfen haben. Unser oben zitierte Gesprächspartner Ahmad kam beispielsweise aus einem Gebiet in Syrien, das der Daesh kontrollierte und berichtete in 
diesem Zusammenhang von vielen traumatischen Erlebnissen. Da die Unterkunft für queere Geflüchtete ebenfalls eine Sammelunterkunft darstellt und daher ähnliche Probleme in Bezug auf Überbelegung und mangelnde Privatsphäre produziert wie konventionelle Unterkünfte (Punkt 3.1), fehlt Geflüchteten mit großen psychischen Problemen, wie Suizidgefährdung, auch hier der adäquate Rückzugsraum, wie Ahmads Beispiel unterstreicht. Nichtsdestotrotz können viele queere Geflüchtete in der Unterkunft der Schwulenberatung ihre queere Lebensweise offener ausleben und werden dafür weniger sozial sanktioniert als in konventionellen Unterkünften. Das monatelange „Verstecken“ der eigenen queeren Lebensweise, was, wie beschrieben, auf einige Interviewpartner*innen extrem negative psychische Effekte hatte, stellt in dieser Unterkunft daher nur für einige queere Geflüchtete ein Problem dar. Die Schilderungen von Aref legen nahe, dass dies insbesondere für Trans*personen zutrifft. Des Weiteren bringen die in Bezug auf queere Lebensweisen geschulten bzw. zum Teil selbst queeren Sozialarbeiter*innen eine höhere Sensibilität für Probleme hinsichtlich der psychischen Gesundheit in Zusammenhang mit queer*feindlichen Diskriminierungen und Übergriffen mit. Auch bieten alle drei, der von uns interviewten Organisationen, selbst psychosoziale Beratung an bzw. vermitteln Betroffene an geeignete Psychotherapeut*innen weiter.

\subsection{Bewertung der Unterkunft}

"It's better than the other places, but anyway, you're still feeling that you don't have everything you want. Because you are still sharing your room, not your flat" (Interview mit Nabil)

Einige der Bewohner*innen bzw. ehemaligen Bewohner*innen der Unterkunft sowie die drei befragten Organisationen werteten es als Fortschritt, dass es diese neue, speziell auf queere Bewohner"innen ausgerichtete Unterkunft gibt. Gleichzeitig unterstrichen die von uns interviewten Organisationen, dass auch diese Unterkunft eine Sammelunterkunft ist und somit ähnliche Problemlagen wie konventionelle Sammelunterkünfte produziere. Darüber hinaus wurde durch die Interviews deutlich, dass marginalisierte Personengruppen innerhalb des queeren Spektrums, wie beispielsweise Trans"personen, besonders feminine schwule Cis-Männer oder nicht-binäre Personen, cisgeschlechtliche Lesben oder bi-sexuelle Personen Diskriminierung bis hin zu physischen Angriffen in der Unterkunft ausgesetzt waren. Somit stellt die queere Unterkunft für sie nur im bedingten Maß bzw. zum Teil auch gar keinen sichereren Wohnort dar. Eine idea- 
le Sammelunterkunft für Geflüchtete würde in den Augen von Hassino Zweibettzimmer für (alle, nicht nur queere) Geflüchtete zur Verfügung stellen. Er wünscht sich darüber hinaus inklusivere Wohnprojekte, in denen nicht nur Geflüchtete miteinander Wohnen. Die Schwulenberatung hat auch ein solches inklusives Wohnprojekt auf den Weg gebracht, in dem potentiell alle Klient"innen der Schwulenberatung, also auch Personen ohne Fluchtgeschichte, wohnen können. Dies würde seiner Ansicht nach eine Segregation von Geflüchteten oder anderen Gruppen verhindern.

\section{Umgangsstrategien mit Unterbringungsverhältnissen in Sammelunterkünften}

"And also in this difficult situation people show immense strength and much, much, much creativity about finding solutions. Maybe to move together with a friend, to get a cheaper flat. And there are also many success stories, where people find solutions." (Interview mit Masha, LesMigraS)

Viele der von uns befragten Geflüchteten empfanden das Leben in einer Sammelunterkunft als stark reguliert und sie berichteten, dass der Handlungsspielraum der Bewohner*innen sehr eingeschränkt werde. Nabil fasste dies folgendermaßen zusammen:

"About nothing you could decide there, because for everything there were rules, you have to do what the rule says. There are so many things that you would like to do, but you could not." (Interview mit Nabil)

Trotz dieser widrigen Umstände entwickelten unsere Interviewpartner*innen durch vielfältige Umgangsstrategien Formen der Agency, um ihre Unterbringungssituation zu verbessern oder zumindest besser ertragen zu können. Neben der Wohnungs- oder WG-Suche, die wir im Folgenden noch erläutern werden, bestand eine Strategie queerer Geflüchteter darin, sich mit den Mitteln, die ihnen zur Verfügung standen, möglichst gemütlich und häuslich einzurichten:

„Even in this very horrible condition [...] people came to my counselling or to some workshops and groups here, and then showed me photos on their mobile phones and said: look, this is how I decorated my room, these are the pictures that I have drawn and put on my walls. Look, we smuggled a microwave from somewhere [...] people are always also having their own agency and own heart. And we are trying to build home even under these unbelievable circumstances. And I think it is also important talking about these whole housing problems and these rigid structures, which are shitty and 
also not forgetting people are trotzdem, despite everything trying to build nice places of it." (Interview mit Masha, LesMigraS)

Des Weiteren versuchten manche queere Geflüchtete in Sammelunterkünften sich durch das Abtrennen ihres Bettes mit Stoff zumindest ein wenig Privatsphäre in Mehrbettzimmern zu schaffen oder verbrachten viel Zeit in ihrem Zimmer, sofern sie ein Einzelzimmer hatten. Andere unserer geflüchteten Gesprächspartner*innen suchten hingegen aktiv den Kontakt $\mathrm{zu}$ anderen Bewohner*innen der Unterkunft und versuchten Freundschaften aufzubauen, um nicht isoliert zu sein. Viele nutzten auch Kontakte zu Freund"innen und zur queeren Community, um Unterstützung, Solidarität oder Ablenkung zu finden. So berichteten mehrere Befragte, dass sie viel Zeit bei ebenfalls geflüchteten Freund*innen verbrachten, die bereits ein eigenes Zimmer oder eine eigene Wohnung hatten. Andere der Interviewten besuchten oft neue Freund*innen, die sie in Deutschland kennengelernt hatten. Unser Interviewpartner Lee freundete sich beispielsweise, während er in einer Kleinstadt wohnte, mit einer deutschen Familie an, bei der er dann die meiste Zeit verbrachte und nach eigener Aussage eine Art Familienmitglied wurde. Andere Umgangsstrategien mit den ungenügenden Unterbringungsbedingungen stellten Zerstreuung durch ausgedehnte Spaziergänge oder kreative Tätigkeiten wie Malen oder Gitarre spielen dar. Zwei Interviewpartner*innen engagierten sich auch ehrenamtlich bei einem sozialen Träger und halfen bei Übersetzungen in den Unterkünften oder bei Behörden. In Ausnahmefällen wendeten einige unserer Gesprächspartner*innen jedoch auch Umgangsstrategien an, die ihnen selbst schadeten. Unser Gesprächspartner Ahmad, der länger in einer Sammelunterkunft gewohnt hatte, schilderte uns beispielsweise, dass er zu dieser Zeit viele Drogen konsumiert hatte, um sich nicht permanent mit seinen schlechten Lebensbedingungen konfrontieren zu müssen. Wieder andere Geflüchtete nahmen schlechte Unterbringungsbedingungen nicht hin und beschwerten sich. Bei Missständen in der Unterkunft wendeten sie sich beispielsweise an die Sozialarbeiter*innen, verfassten Beschwerdebriefe an den Träger, oder trafen sich, um gemeinsam über die Probleme in der Unterkunft zu beraten. Manche Geflüchtete, die gegen ihren Willen in ein anderes Bundeland verlegt wurden, registrierten sich auch erneut unter leicht abgewandeltem Namen ${ }^{22}$, um in einer größeren Stadt bleiben zu können oder zogen bereits vor der Bewilligung des Umzugs um. Un-

22 Die Geflüchteten versuchten damit die Verlegung in ein anderes Bundesland zu umgehen. Mittlerweile werden bereits bei der Registrierung von Geflüchteten Fingerabdrücke von ihnen genommen, weshalb eine zweite Antragsstellung un- 
ser*e Gesprächspartner*in von GLADT erwähnte darüber hinaus Versuche von queeren Geflüchteten, sich beim Auszug aus der Sammelunterkunft gegenseitig zu unterstützen: In den Räumlichkeiten von GLADT hatten sich eine Zeit lang geflüchtete Trans*personen getroffen, um sich bei der Wohnungssuche gegenseitig zu helfen.

\section{Wohnungssuche queerer Geflüchteter}

"It's the housing problem that everybody faces in Berlin, but you can multiply it by ten at least for the refugees [....]. [For] LGBT refugees, [...] accommodation and housing are the biggest issues. Once they are solved, people can start to stabilize.” (Interview mit Mahmoud Hassino, Schwulenberatung)

Eine der bereits erwähnten Umgangsstrategien queerer Geflüchteter, um ihre Wohnsituation zu verbessern, ist das Suchen nach einer eigenen Wohnmöglichkeit. So merkte unser*e Gesprächspartner*in von GLADT an, dass viele queere Geflüchtete eigentlich gar keine Probleme bezüglich ihrer sexuellen Orientierung und/oder Geschlechtsidentität hätten und daher auch keiner Betreuung durch spezifische Stellen bedürften. Die meisten benötigten einfach ihren eigenen Wohnraum und sobald sie diesen erhalten hätten, könnten sie ihr Leben selbstständig meistern. Laut Hassino von der Schwulenberatung hätten viele queere Geflüchtete das Bedürfnis in WGs zu wohnen, bzw. zusammen mit anderen Geflüchteten eine WG zu gründen. Manche unserer geflüchteten Gesprächspartner*innen wollten am liebsten allein wohnen. Unser*e Gesprächspartner*in von GLADT berichtete, dass einige ihrer*seiner Klient*innen, die zwischen 2015 und 2016 nach Deutschland gekommen waren, mittlerweile eine eigene Wohnung gefunden hätten, viele jedoch immer noch auf Wohnungssuche seien. Auch in den von uns geführten Interviews berichteten viele geflüchtete Gesprächspartner*innen, dass sie seit langem auf Wohnungssuche seien. Einige hatten auch nach zwei Jahren in Berlin und großen dahingehenden Bemühungen immer noch keine eigene Wohnung bzw. ein WG-Zimmer gefunden. Sie schätzten es als sehr schwierig ein, für sich in Berlin einen Wohnraum zu finden. Unsere Gesprächspartner*innen definierten verschiedene Faktoren als hemmend bzw. förderlich für die

ter einem anderen Namen nicht mehr möglich ist (vgl. Bundesamt für Migration und Flüchtlinge 2019). 
Wohnungssuche queerer Geflüchteter, die wir im Folgenden zusammenfassen möchten.

\subsection{Berliner Wohnungsmarkt}

„Ich gehe zu einem Besichtigungstermin und dann sehen sie, er ist ein Mann. Und in meinem Ausweis steht Frau. [...] Das ist ein großes Problem. [...] Das BAMF hat mir gesagt, du musst 2.000 bezablen für die Änderung von Geschlecht und Namen, 2.000!" (Interview mit Aref)

Ein Grund dafür, dass viele queere Geflüchtete Probleme haben eine eigene Wohnung oder ein WG-Zimmer zu finden, liegt in der derzeitigen Situation des Berliner Wohnungsmarktes, seiner Strukturen und dem Handeln seiner Akteur*innen. In Berlin herrscht ein starker Wohnraummangel, insbesondere an preiswerten Mietwohnungen. In Berlin fehlten 2018 schon ca. 310.000 Wohnungen im preiswerten Mietsegment (Holm, Lebuhn, Junker und Neitzel 2018: 10). 2016 bezifferte Andrej Holm den Bedarf an preiswerten Wohnungen für Geflüchtete auf ca. 25.000 (Holm 2016: 4). Bezahlbare freie Wohnungen stehen darüber hinaus immer öfter nur in den Berliner Randgebieten zur Verfügung (ebd.: 61). Laut Masha von LesMigraS sei die Akzeptanz gegenüber queeren Geflüchteten hier jedoch zum Teil geringer als in den Berliner Innenstadtgebieten. Außerdem gibt es in den Randgebieten oft keine queere Infrastruktur. So müssen oft lange Fahrtwege zurückgelegt werden, um zu queeren Beratungsstellen oder Lokalitäten zu gelangen (Interview mit Masha, LesMigraS).

Hinzu kommen diskriminierende Strukturen des Wohnungsmarktes, die queere Geflüchtete in ihren Überschneidungen besonders treffen. So liegen bereits Forschungsarbeiten zur Diskriminierung Geflüchteter auf dem Wohnungsmarkt (vgl. Foroutan, Hamann, El-Kayed und Jorek/ BIM 2017b), zu rassistischen Diskriminierungen bei der Wohnungssuche (vgl. Senatsverwaltung für Integration, Arbeit und Soziales 2010) sowie zu Diskriminierungen queerer Mieter*innen durch Vermieter*innen (vgl. Droste/Diesenreiter 2019) vor. Besonders geflüchtete Trans*personen erleben Probleme und Diskriminierung bei der Wohnungssuche, insbesondere wenn ihre Personenstandsänderung noch nicht vollzogen ist, wie das einleitende Zitat dieses Abschnitts verdeutlicht. 


\subsection{Institutionelle Rahmendbedingungen und Zusammenarbeit mit Behörden}

"I really have Glück, [lacht] because the social worker in Sozialamt, they was nice with me, and they told me, you can stay in this private place, and you don't need to stay all the time in the Heim. And in this moment too many people came to Brandenburg, too. And they wanted as much people as possible to move out from there, this Heim." (Interview mit Tatjana)

Neben den Hemmnissen, die durch die Situation des Wohnungsmarktes und seine zum Teil diskriminierenden Strukturen und Akteur"innen bedingt sind, erleben Geflüchtete generell aufgrund ihres Status als Geflüchtete institutionelle Hürden. Hassino bemängelte, dass in seinen Augen insgesamt ein staatlicher Plan fehle, wie Geflüchtete dabei unterstützt werden könnten, von den Sammelunterkünften in private Wohnungen zu ziehen. Schweden, Norwegen oder die Niederlande übernähmen vergleichsweise mehr Verantwortung in diesem Prozess. Laut Einschätzung von Hassino wären bürokratische Prozesse zur Beantragung von Leistungen für Geflüchtete in Deutschland zudem oft langwierig, was insbesondere im Punkto Wohnen ein Problem darstelle. Selbst wenn Geflüchtete eine Zusage für eine Wohnung durch Vermieter*innen erhalten haben, dauere es oft mehrere Wochen, bis sie die Zusage der Mietkostenübernahme durch das Jobcenter erhielten. In der Zwischenzeit werde die Wohnung oder das WG-Zimmer oftmals an jemand anderen vergeben. Auch in Bezug auf die Sprache gebe es oft Komplikationen mit dem Jobcenter. So würden Geflüchtete beispielsweise beim Ausfüllen der deutschsprachigen Formulare nicht unterstützt und Übersetzungen der Formulare seien nicht vorhanden. Insgesamt fehlte es laut Hassino bei den Behörden vor allem an Flexibilität und der Bereitschaft ihren Ermessensspielraum zugunsten der Geflüchteten auszunutzen.

Diese für alle Geflüchteten geltenden Hemmnisse treffen queere Geflüchtete in besonderem Maße, da sie, wie geschildert, durch die Unterbringung in Sammelunterkünften oft mit spezifischen Problemlagen konfrontiert sind. ${ }^{23}$ Queeren Geflüchteten (wie auch nicht queeren Geflüchteten) kann schon vor Ablauf der maximalen zeitlichen Wohnverpflichtung in einer EAE ermöglicht werden, in einer privaten Unterkunft $\mathrm{zu}$ wohnen.

23 Daneben wurde, wie uns in Interviews berichtet wurde, queeren Geflüchteten die Zusammenarbeit mit Behörden allgemein zum Teil auch zusätzlich durch Sprachmittler*innen erschwert, die entweder nicht über die genügende Kompetenz oder Sensibilität in der Übersetzung queerer Thematiken besitzen oder selbst queer*feindlich handeln (vgl. Hassino 2017). 
Dies war bei queeren Geflüchteten 2017 insbesondere durch die erwähnte, bereits 2015 erfolgte, Definition der Schutzbedürftigkeit im erweiterten Sinne durch den Berliner Senat gerechtfertigt. Nach Berichten unserer Interviewpartner*innen sah die gängige behördliche Praxis 2017 jedoch so aus, dass queeren Geflüchteten, die früher als sechs Monate nach Stellung ihres Asylantrages eine Wohnung gefunden hatten, der Umzug in der Regel nicht bewilligt wurde. Selbst bei Freund*innen oder Familie unterzukommen würde in den ersten sechs Monaten in der Regel abgelehnt werden (Interview mit Mahmoud Hassino, Schwulenberatung). Darüber hinaus würden Wohnungen bzw. Zimmer für queere Geflüchtete zum Teil abgelehnt, wenn sie minimal über dem veranschlagten Höchstsatz lägen. Dies sei absurd, da stattdessen ein Platz in einer Unterbringung finanziert würde, der monatlich über 1000 Euro koste und somit deutlich teurer sei (ebd.). Wie das einleitende Zitat dieses Abschnitts zeigt, können Sachbearbeiter*innen, die ihre Ermessenspielräume großzügig ausnutzen, jedoch eine sehr positive Auswirkung auf das Befinden von queeren Geflüchteten haben. Des Weiteren bemängelte unser*e Gesprächspartner*in von GLADT, dass zumindest bis 2017 für queere Geflüchtete bezüglich der Wohnungssuche keine behördlich-institutionelle Unterstützung bzw. Anlaufstelle vorhanden sei. Unterstützung bei der Wohnungsfindung für queere Geflüchtete sei jedoch keine Aufgabe für Ehrenamtliche oder die queere Community, sondern eine staatliche Aufgabe. ${ }^{24}$ Darüber hinaus verwies Masha von LesMigraS auf den Umstand, dass queere Geflüchtete und andere vulnerable Gruppen einen bevorzugten Zugang zu Wohnungen der öffentlichen Hand haben sollten. Es existiert beispielsweise bereits das wohnungspolitische Instrument des Wohnungsberechtigungsscheins (WBS), den man benötigt, um vergünstigte Sozialwohnungen der öffentlichen Hand mieten zu können. Zwar haben anerkannte Geflüchtete (queere wie nicht-queere) theoretisch einen Anspruch auf einen solchen WBS, wenn ihr Aufenthaltstitel noch mindestens 11 Monate gültig ist. Allerdings bemängeln mehrere Berliner Geflüchteten-Organisationen eine restriktive Handhabung der dahingehenden Richtlinien durch die Berliner Bezirke. Des Weiteren kritisieren sie die Beschränkung der Ausstellung des WBS auf solche Geflüchtete, die einen noch mindestens 11-monatigen Aufenthaltstitel besitzen, da dies die oft positiven Bleibeaussichten von Geflüchteten nicht mit einkalkuliert und somit den Zugang von Geflüch-

24 Im September 2020 nahm ein Pilotprojekt zur Wohnraumaquise für Geflüchtete in Berlin seine Arbeit auf. Das Projekt wendet sich dabei jedoch in erster Linie an Vermieter*innen (Senatsverwaltung für Integration, Arbeit und Soziales 2020). 
teten zu preiswertem Wohnraum erschwert (Flüchtlingsrat Berlin et al. 2019).

\subsection{Sprachkenntnisse, Kontextwissen und Ressourcen}

"I really don't know who can help me with a flat, who I can ask about belping me to find a flat, and I have a little bit of fear about that, I really want to have my own [flat] [...] I cannot speak German very well, and this can be a big problem. And I need help from a social worker all the time because I cannot do anything by myself." (Interview mit Kim)

Neben den geschilderten institutionellen Hemmnissen erleben viele Geflüchtete auch durch ihre oft noch eingeschränkten Deutschkenntnisse und ihr mangelndes Kontextwissen zur Wohnungssuche in Deutschland Nachteile. Insgesamt wünschten sich viele der von uns interviewten Geflüchteten daher eine bessere Unterstützung bei der Wohnungssuche, worauf wir im folgenden Abschnitt noch genauer eingehen werden. Unser*e Gesprächspartner*in von GLADT betonte außerdem, dass der Erfolg, den queere Geflüchtete bei der Wohnungssuche haben, auch mit anderen Ressourcen oder Hemmnissen zusammenhängen würden, die queere Geflüchtete ihrerseits mitbrächten. Dazu zählten Faktoren wie Herkunft, Bildungsgrad oder psychische Erkrankungen:

„Das spielt alles mit. Leute, die halt eher Sachen in die Hand nehmen können, haben auch was gefunden, oder wenigstens irgendwelche Wege gefunden. Aber die Menschen, [...] denen einfach andere Voraussetzungen feblen, sind immer noch in einer Unterkunft, oder irgendwo in einer nicht schönen Situation." (Interview mit GLADT)

\subsection{Unterstützung durch Community-Organisationen}

"Schwulenberatung, they are, as I told you, supporting us with things like translating [...] But the main problem for all the gay refugees [are] the houses. They can't do it, they cannot arrange. Because they also need support [...] from some other people or from other organizations to find houses for us [...]. Because in [...] Schwulenberatung, there are working three or four persons, they are doing all the work alone." (Interview mit Jamal)

Unsere Gesprächspartner*innen aus den drei Community-Organisationen betonten, dass sie zwar auf Nachfrage Basisinformationen zur Wohnungs- 
suche bereitstellen würden, dass Unterstützung bei der Wohnungssuche jedoch nicht zu ihren Aufgaben gehöre. Es sprenge ihre Kapazitäten, Geflüchtete dabei aktiv zu begleiten. Diese Einschätzung bestätigten auch von uns interviewte Geflüchtete: Viele berichteten davon, nach ihrer Ankunft in Deutschland bei Berliner queeren Community-Organisationen Rat und Unterstützung gefunden zu haben. Das Beratungsangebot bezog sich jedoch in der Regel nicht auf Fragen der Unterkunft.

Das einzige Projekt in Berlin, in dessen Rahmen nach Aussage der von uns interviewten Organisationen hierzu ein spezielles Angebot geschaffen wurde, war das Programm SAWA, das von GLADT initiiert worden war und 2016 queere Geflüchtete bei der Wohnungssuche unterstützte. Das Projekt wurde 2017 jedoch wegen mangelnder Förderung eingestellt. Zum Zeitpunkt der Interviews war es unklar, ob dieses oder ein ähnliches Projekt in den kommenden Jahren wieder gefördert werden würde. Allerdings schätzten unsere Interviewpartner*innen von GLADT und der Schwulenberatung das Projekt auch angesichts des großen Bedarfs als zu klein ein, um eine wirklich wirksame Unterstützung von queeren Geflüchteten bei der Wohnungssuche oder -vermittlung gewährleisten zu können.

Der Lesben- und Schwulenverband in Deutschland (LSVD) hatte einigen unserer Interviewpartner*innen ebenfalls Unterstützung bei der Wohnungssuche angeboten, wobei die Erfahrungen mit diesem Programm gemischter Natur waren. Einige der externen Berater*innen oder Hauptmieter"innen, die der LSVD vermittelt hatte, äußerten sich rassistisch oder versuchten ihre Position dafür auszunutzen sexuelle Beziehungen zu den Geflüchteten aufzubauen. Andere Geflüchtete haben jedoch auch gute Erfahrungen mit Vermittlungen durch den LSVD gemacht und so eine WG gefunden, in der sie sich wohl fühlten. Es scheint gerade bei der Weitervermittlung an externe Personen zur Unterstützung queerer Geflüchteter wichtig zu sein, dass diese Personen in Bezug auf Mehrfachdiskriminierungen geschult werden und dass Hinweisen auf diskriminierendes Verhalten oder sexualisierte Gewalt konsequent nachgegangen wird. Masha von LesMigraS hob darüber hinaus die Bedeutung und den Nutzen von Organisationen hervor, die kontinuierlich und langfristig zu queeren Themen, Flucht und Mehrfachdiskriminierung arbeiten, wie LesMigraS oder GLADT. Dort sei eine spezifische Expertise vorhanden, über die viele Organisationen, die bisher hauptsächlich zu queeren Themen gearbeitet 
haben, nicht verfügen, die jetzt aber dringend und schnell gebraucht werde. ${ }^{25}$

Mehrere Geflüchtete berichteten darüber hinaus auch von positiven Erfahrungen mit einer arabischen Community-Organisation ${ }^{26}$ in Neukölln, die Beratung für Geflüchtete anbietet. Dort war einem Gesprächspartner ein Rechtsanwalt vermittelt worden, mit dessen Hilfe er gerichtlich seinen Umzug nach Berlin durchsetzen konnte und der ihn an die Schwulenberatung weiter verwies. In einem anderen Fall unterstützte diese Organisation eine geflüchtete Person bei der Beantragung von Geldern für eine eigene Wohnung beim Jobcenter.

\section{Queere Geflüchtete in privaten Wohnverhältnissen}

"When I ran away from the Middle East, I was thinking I will finally have a stable life, there are at least no problems [...] with the house. I couldn't imagine that I will find these problems here in Germany. So, when I saw the situation [...] of the house, I felt very bad. Sometimes I ask myself what I did to myself, where I brought myself." (Interview mit Jamal)

Nachdem wir im vorherigen Abschnitt die Schwierigkeiten von queeren Geflüchteten bei der Wohnungssuche geschildert haben, möchten wir nun auf die Wohnsituationen queerer Geflüchteter in privaten Wohnformen eingehen, denn viele unserer geflüchteten Gesprächspartner*innen sahen sich auch nach ihrem Auszug aus der Sammelunterkunft mit Problemen in ihren Wohnverhältnissen konfrontiert. Private Wohnverhältnisse sind beispielsweise das Wohnen in WGs, mit Partner*innen oder bei Verwandten. Keine der von uns interviewten geflüchteten Personen hatte eine eigene Wohnung, die sie allein bewohnte. Als Missstände in privaten Wohnkonstellationen identifizierten unsere Interviewpartner*innen Probleme mit Mitbewohner*innen aufgrund von rassistischem und/oder queer*feindlichem Verhalten oder sexualisierter Gewalt, Angst sich vor Verwandten zu outen oder prekäre und beengte Wohnverhältnisse.

25 Vertiefend zu Standards in der professionellen sowie der ehrenamtlichen Arbeit und Beratung mit queeren Geflüchteten sind die Artikel „Spezifika in der Beratung von LSBT"I* Geflüchteten“ von Masha Beketova (2017) sowie „Ehrenamtsmanagement im LSBT*T*I-Bereich" von Gesa Luise Rittinghaus (2017).

26 An den genauen Namen der Organisation konnten sich die Befragten nicht erinnern. 
Einige Geflüchtete, die wir befragten, hatten, nachdem sie einige Zeit in einer Sammelunterkunft gelebt hatten, einen Platz in einer WG gefunden, oft bei Personen aus der queeren Community in Berlin. Zum Teil waren jedoch die Machtbeziehungen, die diesen Wohnungssituationen zugrunde lagen, ein Problem. Geflüchtete wurden in der Regel in bestehende WGs oder auch Wohnungen von Einzelpersonen aufgenommen. In manchen Wohnkonstellationen sahen sich die Geflüchteten jedoch aufgrund von Vorurteilen oder rassistischen Einstellungen seitens der Hauptmieter*innen erneut mit Diskriminierungen konfrontiert:

„Even people who manage to find housing [...] living with a German [...] sometimes what happens is that [...] the person who is offering a room in their flats, come from this white privileged, white supremacy idea: I want to help this person to integrate, so this is how it's done. And once, they face [...] they are not getting the outcome they wanted [...] they become racist." (Interview mit Mahmoud Hassino, Schwulenberatung)

Unser Gesprächspartner Jamal berichtete von solch rassistischer Bevormundung durch seinen Hauptmieter:

"I feel bad in my house [...] when we fight. We are fighting because of him, he wants me to sleep at his time, he wants me to wake up at his time, I cannot do that. I have no work, I have no school, nothing to do [...]. He wants me to make my life the same as his life. So we are fighting." (Interview mit Jamal)

Ein weiteres Problem resultiert aus dem Verhalten von Hauptmieter"innen, die ihre Machtposition als Halter*innen der Wohnung ausnutzten und sexualisierte Gewalt gegenüber den mit ihnen lebenden Geflüchteten ausüben. Jamal berichtete von solch einer gewaltvollen Wohnsituation. Da er jedoch keine alternative Wohnmöglichkeit hatte und unter keinen Umständen zurück in eine Sammelunterkunft wollte, verblieb er in diesem gewaltvollen Wohnkontext. Wieder andere Gesprächspartner*innen waren mit homo"feindlichen Mitbewohner"innen konfrontiert. Sam berichtete beispielsweise, dass er mit zwei heterosexuellen Männern zusammenlebte, mit denen er sich zunächst gut verstanden hatte. Nachdem sie jedoch erfuhren, dass er schwul ist, verschlechterte sich ihr Verhältnis und sie mieden ihn. Auch das zeitweise Wohnen bei Verwandten war für einige, jedoch nicht alle, unserer Interviewpartner*innen problematisch. Unser Interviewpartner Jamie, der ein halbes Jahr lang im Hause seines Onkels gewohnt hatte, hatte dort beispielsweise noch viel mehr Angst als in der Sammelunterkunft, dass jemand herausfinden könnte, dass er schwul ist, 
da er davon ausging, dass seine Verwandten dies nicht akzeptieren würden.

Neben Problemen mit Mitbewohner*innen oder Verwandten stellten auch außerhalb von Sammelunterkünften prekäre oder beengte Wohnverhältnisse ein Problem für unsere Gesprächspartner*innen dar. Nabil berichtete, dass er nur ein auf wenige Monate befristetes Zimmer gefunden hatte; Sam, dass er mit anderen Untermieter*innen zusammenwohnte, die er sich nicht selbst ausgesucht hatte und die ständig durch den Hauptmieter ausgetauscht würden. Unser Gesprächspartner Jamie bemängelte, dass er in Berlin bereits sechsmal von einer kurzfristigen Unterkunft zur nächsten umgezogen sei. Unser"e Interviewpartner*in Nur schilderte, er"sie hatte über den LSVD eine WG gefunden und war dort zunächst mit zwei Bekannten, mit denen er*sie zusammen geflohen war, eingezogen. Sie teilten sich drei Monate lang ein sehr kleines Zimmer und hatten daher viele Konflikte. Insgesamt hatten nur fünf der zwölf von uns interviewten geflüchteten Personen mittlerweile eine Wohnsituation gefunden, in der sie sich wohl fühlten, eine Person davon hatte jedoch nur einen kurzzeitigen Mietvertrag für ein paar Monate. Vier dieser Personen wohnten in WGs, eine Person mit dem Partner. Dabei berichteten diese Personen, wie sehr sie ihre jetzige Wohnsituation positiv beeinflusste:

"It's a really big difference, when you have your place, when you are outside, and you come back to your place, so you say, ah, finally, like, I have my place. Because it's a dream in Berlin to get a place. And I really feel so comfortable in my place, and I would like to stay, because I have the best amazing location and a view. So, when you have your place, you feel you don't need anything else, it's everything I have right now. Because it was so hard to get your place." (Interview mit Nabil)

Unser Interviewpartner Lee schilderte darüber hinaus er habe nun, da er sein eigenes Zimmer bewohnte, zum ersten Mal seit seiner Ankunft in Deutschland das Gefühl, dass er wieder „er selbst" sein könne. Diese Äußerungen unserer geflüchteten Gesprächspartner*innen verweisen zum Abschluss dieses Abschnitts auf die besondere Bedeutung, die ein eigener, stabiler Wohnraum für queere Geflüchtete hat. Im Folgenden möchten wir nun die Ergebnisse unserer Kurzstudie zusammenfassen, einige Empfehlungen zu Verbesserungsmöglichkeiten in den Wohnsituationen queerer Geflüchteter herausarbeiten und mit einem Ausblick zum Themenkomplex schließen. 


\section{Fazit und Empfehlungen zur Unterbringung queerer Geflüchteter}

„[H]aving your own place [...] gives you all the control, you know?" (Interview mit Nur)

Die Erfahrungen unserer geflüchteten Gesprächspartner*innen sowie unserer Befragten von den drei Organisationen LesMigraS, GLADT und der Schwulenberatung Berlin zu den Wohn- und Unterbringungssituationen queerer Geflüchteter machen deutlich, dass insbesondere die dauerhafte Sammelunterbringung von Geflüchteten problematische Wohn- und Lebensverhältnisse produziert. Wie gezeigt wurde, haben dabei einerseits die Dimensionen Sicherheit, sozialarbeiterische Betreuung, Auslastung und Privatsphäre in sowie die Lage von Sammelunterkünften und andererseits die psychische Gesundheit queerer Geflüchteter eine besondere Bedeutung. Das bedeutet selbstverständlich nicht, dass diese Aspekte für nicht-queere Geflüchtete unwichtig sind oder sie nicht auch unter den mangelhaften Bedingungen entlang dieser Dimensionen in Sammelunterbringungen leiden. Jedoch weisen die Ergebnisse unserer Kurzstudie darauf hin, dass sich für queere Geflüchtete entlang dieser Dimensionen spezielle Problemlagen ergeben. Diese variieren je nach spezifischer sozialer Positionierung ${ }^{27}$ und damit verbundenen Ressourcen, da queere Geflüchtete eine diverse Gruppe darstellen. Spezielle Sammelunterkünfte für queere Geflüchtete sind daher zwar in einigen Punkten ein Fortschritt, da hier eine Sensibilisierung für queere Lebensweisen vorhanden ist, sie erzeugen jedoch, wie deutlich wurde, ähnliche Problemlagen wie konventionelle Sammelunterkünfte. Sammelunterbringung ist weder für queere noch für nicht-queere Geflüchtete eine bedarfsgerechte Form der Unterbringung. Tuğba Tanyılmaz, Nadiye Ünsal und Koray Yılmaz-Günay (2019) unterstreichen in diesem Zusammenhang, dass ein „Exzeptionalismus, der einzelnen Gruppen von Geflüchteten ausnahmsweise bessere Lebensbedingungen gewährleistet, auch dazu dient, systematisch schlechtere

27 Queere Geflüchtete sind, wie alle Individuen, auf verschiedenen Ebenen sozial intersektional positioniert. So nehmen sie beispielsweise in Bezug auf die Dimensionen Alter, Klassenzugehörigkeit, Trans*-/ oder Cisgeschlechtlichkeit, spezifische Art der Rassifizierung oder auch entlang der Dimension abled/disabled unterschiedliche Positionen ein. Diese sozialen Positionierungen überschneiden sich auf verschiedene Arten und Weisen und schaffen so spezifische Diskriminierungs- oder auch Privilegierungserfahrungen. 
Lebensbedingungen für andere zu legitimieren“ (ebd.: 137). ${ }^{28}$ Aufgrund dessen empfehlen wir: ${ }^{29}$

\section{Dezentrale Wohnunterbringung für alle Geflüchteten}

Allen Geflüchteten muss es ermöglicht werden, in dezentralen Unterbringungsformen zu leben. Die Unterbringungsdauer in Sammelunterkünften muss auf das absolute Minimum reduziert werden, die Handlungsmacht und Wahlmöglichkeiten queerer und nicht-queerer Geflüchteter in Bezug auf Wohnen müssen gestärkt werden. Geflüchtete im Allgemeinen und queere Geflüchtete im Besonderen dürfen nicht gezwungen sein, in peripheren Unterkünften leben zu müssen. Für viele queere Geflüchtete ist es wichtig, in einer Großstadt leben zu können, in der sie Anschluss an eine queere Community und Infrastruktur finden können. Geflüchteten sollte es freistehen, sich einen Wohnort innerhalb Deutschlands auszusuchen. Sofern Geflüchtete in WGs zusammenleben, ist es wichtig, dass sie frei entscheiden können, mit wem sie zusammenwohnen. Eine starke Abhängigkeit von Hauptmieter*innen muss vorgebeugt werden, damit queere Geflüchtete nicht aus Mangel an Alternativen in gewaltvollen Wohnkontexten verbleiben. Es könnte ebenfalls ratsam sein, für (potenzielle) Mitbewohner*innen queerer Geflüchteter Workshops zur Sensibilisierung in Bezug auf Mehrfachdiskriminierungen und die damit einhergehenden Machtverhältnisse anzubieten.

28 Sie sprechen sich daher generell gegen die Einrichtung spezieller Unterkünfte für queere Geflüchtete oder Frauen* aus. Stattdessen plädieren sie für einen Verzicht auf „Ausnahmen von der Regel, denen es ein bisschen besser gehen soll“ (ebd.: 148) zugunsten einer Erweiterung der Perspektive, die die Abschaffung der Dublin-Verordnung, der Ermöglichung von Bewegungs- und Niederlassungsfreiheit sowie die Abschaffung des Asylbewerberleistungsgesetzes und eine Gleichbehandlung nach Sozialgesetzbuch für alle Geflüchteten fordert (ebd.: f.).

29 Unsere Empfehlungen decken sich zum Teil mit den Empfehlungen zur Umsetzung der Mindeststandards für LSBTI* Geflüchtete im Annex der „Mindeststandards zur Unterbringung von geflüchteten Menschen in Flüchtlingsunterkünften“ des Bundesministeriums für Familie, Senioren, Frauen und Jugend (BMFSFJ) und UNICEF (2018). Die darin enthaltenen Empfehlungen für queere Geflüchtete wurden von der Schwulenberatung Berlin koordiniert und redaktionell betreut. 


\section{Spezifische Anforderungen bei der temporären Unterbringung queerer Geflüchteter in Sammelunterkünften}

Benötigt werden spezifische Unterkünfte für queere Geflüchtete als Überbrückungslösung, solange flächendeckend keine dezentrale Unterbringung gewährleistet werden kann. Diese können jedoch nur Übergangslösungen sein und ersetzen keine flächendeckende dezentrale Unterbringung für alle Geflüchteten. Während der Unterbringung in einer queeren Sammelunterkunft, muss Privatsphäre ermöglicht werden. Dazu sollten deutlich weniger Personen pro Zimmer aufgenommen werden. Kann geflüchteten Queers kein Platz in einer queeren Unterkunft angeboten werden, müssen für queere Geflüchtete zumindest Einzelzimmer bereitgestellt werden. ${ }^{30}$ Darüber hinaus muss für Schutz und Betreuung besonders vulnerabler Gruppen innerhalb von queeren Sammelunterkünften gesorgt werden. So sollten Trans*geflüchtete oder auch cisgeschlechtliche lesbische Geflüchtete, soweit möglich, keine Wohnung mit cisgeschlechtlichen Männern teilen müssen, wenn sie dies ablehnen. Des Weiteren muss insbesondere für Trans*personen die Möglichkeit bestehen, einen abschließbaren und im Idealfall nicht nach Geschlechtern getrennten Sanitärbereich zu nutzen (vgl. BMFSFJ/ UNICEF 2018: 37). Einer Diskriminierung insbesondere von Trans*personen muss insgesamt aktiv durch das Personal begegnet werden. Kann ein diskriminierungsarmer Wohnkontext in der Sammelunterkunft für Trans*personen nicht gewährleistet werden, müssen Trans*personen besonders bei der Wohnungssuche unterstützt werden. Die von LesMigraS und der Schwulenberatung bereits durchgeführten Sensibilisierungsworkshops für Personal von Sammelunterkünften sollten regelmäßig in allen Unterkünften (queeren wie nicht-queeren) durchgeführt werden. Wenn möglich, sollten auch genügend Sozialarbeiter*innen, die selbst queer und/oder trans* sind, eingestellt werden bzw. alle Sozialarbeiter*innen für die Bedürfnisse dieser Gruppen sensibilisiert werden. Schließlich müssen queere Partner*innenschaften bei der Unterbringung berücksichtigt werden. Queere Paare sollten unabhängig von ihrem Familienstand die Möglichkeit erhalten, zusammen zu wohnen und sollten nicht auf verschiedene Unterkünfte in ggf. unterschiedlichen Bundesländern verteilt werden (vgl. BMFSFJ/ UNICEF 2018: 37).

30 Hier ist darauf hinzuweisen, dass für queere Geflüchtete, die sich nicht outen möchten, eine bevorzugte Behandlung zum Teil auch schwierig sein kann, da sie dadurch ggf. geoutet werden könnten. Die dahingehenden Wünsche der geflüchteten Person sollten hier im Vordergrund stehen. 


\section{Mehr Unterstützung bei der Wohnungssuche für alle Geflüchteten}

Neben den Wohn- und Unterbringungssituationen queerer Geflüchteter haben wir ebenfalls die Erfahrungen mit Behörden und Community-Organisationen bezüglich der Wohnungssuche abgefragt. Daraus ergibt sich unsere dritte Empfehlung. Das von GLADT initiierte Projekt $S A W A$ zur Unterstützung queerer Geflüchteter bei der Wohnungssuche wies in die richtige Richtung, war jedoch nach Aussagen unserer Interviewpartner*innen mit zu wenigen Ressourcen ausgestattet, um bei der Wohnungssuche für queere Geflüchtete wirkliche Erfolge erzielen zu können. In diesem Bereich bedarf es einer langfristigen und stabilen Förderung von Projekten, die staatlich finanziert werden. Zudem sollten Angebote für queere Geflüchtete zur Wohnungssuche in einem für Mehrfachdiskriminierungen sensibilisierten Setting stattfinden. Organisationen sollten sowohl ihre Beratenden als auch ggf. eingesetzte Freiwillige dahingehend schulen. Wenn Organisationen als Vermittler*innen zwischen queeren Geflüchteten und Hauptmieter*innen oder freiwilligen Mentor*innen auftreten, sollten sie Hinweisen auf diskriminierendes Verhalten und sexualisierte Gewalt konsequent nachgehen und diese Personen aus ihren Unterstützungsstrukturen ausschließen. Für Freiwillige ist eine Schulung vor Aufnahme ihrer Tätigkeit notwendig. Ferner sollten Behörden bei der Bewilligung von Leistungen in Bezug auf Wohnunterbringung für alle Geflüchtete ihren Ermessensspielraum voll ausnutzen. Bewilligungsanträge für Wohnungen oder WG-Zimmer sollten so schnell wie möglich bearbeitet werden, um zu verhindern, dass Vermieter*innen die Wohnmöglichkeit anderweitig vergeben. Ebenso sollten alle Personen mit Status als Geflüchtete unabhängig von der Restlaufzeit ihres Aufenthaltstitels Anrecht auf einen WBS haben. Die von den interviewten Organisationen bereits durchgeführten Sensibilisierungsworkshops für Personal von Sammelunterkünften sind auch für das Personal von Behörden, die mit queeren Geflüchteten Kontakt haben, zu empfehlen (vgl. BMFSFJ/ UNICEF 2018: 35). Auch kommunale Wohnungsbaugesellschaften oder private Träger"innen der Wohnungswirtschaft könnten von solchen Sensibilisierungsworkshops profitieren, um Diskriminierungen (nicht nur) von queeren Geflüchteten bei der Wohnungssuche vorzubeugen.

\section{Ausblick}

Der Mietendeckel in Berlin hatte ersten Evaluierungen zufolge zwar zu sinkenden Mieten in dem durch ihn regulierten Mietsegment geführt 
(Immoscout24.de 2021). Er wurde jedoch am 15.04.2021 durch das Verfassungsgericht gekippt, da nach dessen Meinung die betreffende Gesetzgebungskompetenz nicht beim Land Berlin, sondern beim Bund liege. Der gescheiterte Mietendeckel wird daher voraussichtlich zu weiter steigenden Mieten in Berlin führen, da die vom Bund 2015 beschlossene Mietpreisbremse eine zwar verlangsamende, jedoch nur geringe und kurzfristige Wirkung auf die Mietpreisentwicklung hat und der Mietanstieg insgesamt dadurch nicht gestoppt wird (vgl. DIW 2018: 40; Breidenbach, Eilers und Fries 2019: 3). Immer mehr Mieter*innen in Berlin haben Probleme eine erschwingliche Wohnung zu finden, was zu einer Erhöhung der Konkurrenz um freie und bezahlbare Mietwohnungen führt. Es ist daher ein generelles Umdenken in der Wohnungspolitik erforderlich, denn Wohnen ist ein Grundrecht, das für alle Personen gelten sollte, und darf keine Ware sein. Die jetzige Wohnungsmarktsituation in Berlin und vielen anderen deutschen Großstädten führt dazu, dass Gruppen mit weniger finanziellen Ressourcen sowie sozialem und kulturellem Kapital, zu denen queere Geflüchtete in unterschiedlichem Maße gehören, immer schwerer bezahlbaren Wohnraum finden, der ihren Bedürfnissen entspricht.

Zwar hat die Anzahl von Asylanträgen von Geflüchteten in Deutschland seit 2016 kontinuierlich abgenommen (vgl. BAMF 2021: 6), was unter anderem der rigiden und zum Teil illegalen Abwehr von Geflüchteten an den EU-Außengrenzen geschuldet ist (vgl. ECCHR 2020: 29-37). Nichtsdestotrotz werden angesichts der internationalen kriegerischen Auseinandersetzungen, zunehmender Naturkatastrophen in Folge des Klimawandels, des internationalen Wohlstandgefälles zwischen globalem Norden und Süden sowie der Verfolgung queerer Personen, (queere) Geflüchtete weiterhin versuchen, in der EU Asyl zu erhalten - und ein Teil von ihnen wird dies trotz der zum Teil mörderischen Grenzabschottungspolitik auch weiterhin schaffen. Unter ihnen werden auch weiterhin queere Geflüchtete sein. Wohnraum für Geflüchtete zu schaffen, ist also ein Vorhaben, das jetzt wie auch langfristig und vorausschauend betrieben werden muss - auch um eine Situation wie von 2015-2018 zu vermeiden, in der die dahingehende staatliche Infrastruktur, die zuvor in langen Jahren abgebaut worden war, mit der Zahl der Geflüchteten komplett überfordert war.

Unsere Kurzstudie hat deutlich gemacht, dass queere Geflüchtete hierbei besondere Bedürfnisse und Bedarfe haben, die sich von der Erstaufnahme bis in den regulären Wohnungsmarkt ziehen. Zwar zeigen queere Geflüchtete zum Teil sehr kreative Umgangsstrategien mit ihren schlechten Wohnbedingungen, Ziel muss es jedoch sein, queeren und allen anderen Geflüchteten so schnell wie möglich eine Wohnform zu ermöglichen, die ihren Bedürfnissen entspricht. Dies ist insbesondere angesichts der 
Auswirkungen der Corona-Pandemie auf die Lebensbedingungen von Geflüchteten, vor allem jenen, die in Sammelunterbringungen leben müssen, mehr als dringlich. ${ }^{31}$

\section{Literatur:}

Alessi, Edward J.; Kahn, Sarilee; Woolner, Leah; Van Der Horn, Rebecca (2018). Traumatic stress among sexual and gender minority refugees from the Middle East, North Africa, and Asia who fled to the European Union. In: Journal of Traumatic Stress, 31(6). S. 805-815.

Bauer, Isabella (2017). Unterbringung von Flüchtlingen in deutschen Kommunen: Konfliktmediation und lokale Beteiligung. Flucht: Forschung und Transfer, Research Paper Nr. 10. Osnabrück: Institut für Migrationsforschung und Interkulturelle Studien (IMIS); Universität Osnabrück / Bonn: Internationales Konversionszentrum Bonn (BICC). Abgerufen am 07.08.2017 unter https://flucht-forsch ung-transfer.de/publikationen/

Beketova, Masha (2017). Spezifika in der Beratung von LSBT"I* Geflüchteten. In: „Wie eine Rose, die aus dem Riss im Beton wächst." Empowerment von lesbischen, schwulen, bisexuellen sowie trans" und inter" Geflüchteten durch Beratung und Unterstützung. Berlin: Schwulenberatung Berlin. S. 16-24. Abgerufen am 28.03.2021 unter https://schwulenberatungberlin.de/post/empowerment-furschwule-lesbische-bisexuelle-sowie-trans-und-inter-gefluchtete-eine-handreichun g-fur-beratung-und-unterstutzung

Bozorgmehr, Kayvan; Hintermeier, Maren; Razum, Oliver; Mohsenpour, Amir; Biddle, Louise; Oertelt-Prigione, Sabine; Spallek, Jakob; Tallarek, Marie; Jahn, Rosa (2020). SARS-CoV-2 in Aufnahmeeinrichtungen und Gemeinschaftsunterkünften für Geflüchtete: Epidemiologische und normativ-rechtliche Aspekte. Version 1.0. Bremen: Kompetenznetz Public Health COVID-19. Abgerufen am 25.11.2020 unter https://www.public-health-covid19.de/images/2020/Ergebnisse/ FactSheet_PHNetwork-Covid19_Aufnahmeeinrichtungen_v1_inkl_ANNEX.pd $\mathrm{f}$

Breidenbach, Philipp; Eilers, Lea; Fries, Jan (2019). Rent Control and Rental Prices: High Expectations, High Effectiveness? Ruhr Economic Papers \#804. Essen: RWI - Leibniz-Institut für Wirtschaftsforschung. Abgerufen am 27.04.2021 unter https:/www.rwi-essen.de/media/content/pages/publikationen/ruhr-economic -papers/rep_19_804.pdf

31 Das Risiko Geflüchteter, sich in Gemeinschaftsunterkünften in Deutschland, in denen es einen Corona-Fall gibt, mit dem SARS-Cov-2-Virus zu infizieren, wird medizinisch als vergleichseweise hoch bewertet, da Maßnahmen zur physischen Distanzierung nicht oder nur sehr bedingt umsetzbar sind. Bozorgmehr et al. empfehlen daher eine dezentrale Unterbringung und lehnen eine Kollektivquarantäne in Sammelunterkünften ab (Bozorgmehr et al. 2020). 
Bundesamt für Migration und Flüchtlinge (2019). Ankunft und Registrierung. Nürnberg: Bundesamt für Migration und Flüchtlinge. Abgerufen am 08.10.2020 unter https:/www.bamf.de/DE/Themen/AsylFluechtlingsschutz/AblaufAsylverf ahrens/AnkunftRegistrierung/ankunftregistrierung-node.html

Bundesamt für Migration und Flüchtlinge (2021). Aktuelle Zahlen: Ausgabe Februar 2021. Nürnberg: Bundesamt für Migration und Flüchtlinge. Abgerufen am 18.03.2021 unter https://www.bamf.de/SharedDocs/Anlagen/DE/Statistik/As ylinZahlen/aktuelle-zahlen-februar-2021.html?nn=284722

Bundesministerium für Familie, Senioren, Frauen und Jugend (BMFSFJ)/ UNICEF (2018). Umsetzung der Mindeststandards für LSBTI* Geflüchtete, koordiniert und redaktionell betreut von der Schwulenberatung Berlin. In: Annex der Mindeststandards zur Unterbringung von geflüchteten Menschen in Flüchtlingsunterkünften. Berlin: BMFSFJ/UNICEF. S. 33-37. Abgerufen am 25.11.2020 unter https:/www.bmfsfj.de/blob/jump/117472/mindeststandards-zum-schutz-von-gef luechteten-menschen-in-fluechtlingsunterkuenften-data.pdf

Christ, Simone; Meininghaus, Esther; Röing, Tim (2017). "All day waiting": Konflikte in Unterkünften für Geflüchtete in NRW. BICC Working Paper Nummer 3/2017. Bonn: Bonn International Center for Conversion (BICC). Abgerufen am 23.03.2021 unter https:/www.bicc.de/publications/publicationpage/publication/ all-day-waiting-konflikte-in-unterkuenften-fuer-gefluechtete-in-nrw-697/

Classen, Georg/ Flüchtlingsrat Berlin (2017): Ratgeber für Geflüchtete in Berlin. 2. Auflage. Berlin: Flüchtlingsrat Berlin. Abgerufen am 18.10.2020 unter http://ww w.fluechtlingsinfo-berlin.de/fr/pdf/ratgeber.pdf

Deery, Claire/ Republikanischer Anwältinnen- und Anwälteverein (2019). Stellungnahme des RAV zur Aufenthaltsdauer in Landesaufnahmeeinrichtungen nach Einführung des Zweiten Gesetzes zur besseren Durchsetzung der Ausreisepflicht („Geordnete-Rückkehr-Gesetz“). Berlin: Republikanischer Anwältinnenund Anwälteverein. Abgerufen am 19.03.2021 unter https://fluechtlingsrat-berli n.de/wp-content/uploads/stn-rav_ausweitungunterbringung-1.pdf

Deutscher Bundesrat (2019). Drucksache 275/1/19. Empfehlungen der Ausschüsse zu Punkt ... der 979. Sitzung des Bundesrates am 28. Juni 2019: Zweites Gesetz zur besseren Durchsetzung der Ausreisepflicht. Köln: Bundesanzeiger. Abgerufen am 19.03.2021 unter https://www.bundesrat.de/SharedDocs/drucksachen/20 19/0201-0300/275-1-19.pdf?_blob=publicationFile\&v=1.

Deutsches Institut für Menschenrechte (2017). Entwicklung der Menschenrechtssituation in Deutschland. Juli 2016 - Juni 2017. Abgerufen am 28.01.2018 unter http://www.institut-fuer- menschenrechte.de/fileadmin/user_upload/Publikationen/Menschenrechtsbericht_2017/Menschenrechtsbericht_2017.pdf. 
Deutsches Institut für Wirtschaftsforschung Berlin e.V. (DIW Berlin) (2018). Evaluierung der Mietpreisbremse. Endbericht: Untersuchung der Wirksamkeit der in 2015 eingeführten Regelungen zur Dämpfung des Mietanstiegs auf angespannten Wohnungsmärkten (Mietpreisbremse). Studie im Auftrag des Bundesministerium der Justiz und für Verbraucherschutz (BMJV). Berlin: DIW. Abgerufen am 27.04.2021 unter https://www.bmjv.de/SharedDocs/Downloads/DE/Minister ium/ForschungUndWissenschaft/MPB_Gutachten_DIW.pdf?_blob=publicatio nFile\&v=2

Deutschlandfunk (2015). „Daesh“ statt „IS“: Begriff für die Terrorgruppe. Köln: Deutschlandradio. Abgerufen am 16.03.2021 unter https://www.deutschlandfun k.de/begriff-fuer-die-terrorgruppe-daesh-statt-is.2852.de.html?dram:article_id=33 7319

Dilger, Hansjörg; Dohrn, Krista (Hrsg.); International Women Space (2016). Living in Refugee Camps in Berlin: Women's Perspectives and Experiences. Berliner Beiträge zur Ethnologie, Bd. 40. Berlin: Weißensee Verlag.

Dissens (2020). Wörterbuch. Berlin: Dissens - Institut für Bildung und Forschung e.V. Abgerufen am 18.10.2020 unter https:/genderdings.de/gender-woerterbuch /.

Dittmer, Cordula; Lorenz, Daniel F. (2016). „Waiting for the bus that never comes": Quick Response Erhebung von Bedürfnissen und Selbsthilfepotenzialen geflüchteter Menschen in einer Berliner Notunterkunft. Berlin: Katastrophenforschungsstelle.

Droste, Christine; Diesenreiter, Carina (2019). Befunde zur Diskriminierung queerer Lebenswelten im Bereich des Wohnens. In: Queer Wohnen in Berlin - diskriminierungsfreie Zugänge fördern, vielfältige Wohnformen sichern. Berlin: Fair mieten - Fair wohnen. Berliner Fachstelle gegen Diskriminierung auf dem Wohnungsmarkt/ Neustart - Beratung und Wohnunterstützung. S. 15-22. Abgerufen am 23.03.2021 unter http://fairmieten-fairwohnen.de/wp-content/uploads/ 2020/07/Broschuere-Queer-Wohnen-Diskriminierungsfreie-Zug\%C3\%A4nge-foe rdern-vielfaeltige-Wohnformen-sichern.pdf

Europäisches Parlament/ Rat der Europäischen Union (2013). Amtsblatt der Europäischen Union: Richtlinie 2013/33/EU des Europäischen Parlaments und des Rates vom 26. Juni 2013 zur Festlegung von Normen für die Aufnahme von Personen, die internationalen Schutz beantragen (Neufassung). Abgerufen am 23.03.2021 unter https://eur-lex.europa.eu/LexUriServ/LexUriServ.do?uri=OJ:L:2 013:180:0096:0116:DE:PDF

European Center for Constitutional and Human Rights (ECCHR) (2020). Mit Recht gegen Europas Grenzregime: Flucht und Migration. In: Jahresbericht des ECCHR. Menschenrechte mit juristischen Mitteln durchsetzen. Berlin: European Center for Constitutional and Human Rights e.V. S. 29-37. Abgerufen am 25.11.2020 unter https://www.ecchr.eu/fileadmin/Jahresberichte/ECCHR_JB_20 19_DE.pdf 
Fischer, Gabriele; Ober, Nadine; Projektgruppe der Hochschule Esslingen (2019). queer und hier: Sensibilisierung von Sozialarbeitenden in Unterkünften. Projektgruppe der Hochschule Esslingen erarbeitet Bildungsmaterial. In: Refugees \& Queers: Forschung und Bildung an der Schnittstelle von LSBTTIQ, Fluchtmigration und Emanzipationspolitiken. Küppers, Carolin / Bundesstiftung Magnus Hirschfeld (Hrsg.). Berlin: transcript. S. 163-172.

Flüchtlingsrat Baden-Württemberg (2020). Besonders schutzbedürftige Flüchtlinge. Stuttgart: Flüchtlingsrat Baden-Württemberg. Abgerufen am 18.10.2020 unter https://aktiv.fluechtlingsrat-bw.de/besonders-schutzbeduerftige-fluechtlinge. html

Flüchtlingsrat Berlin e.V.; offensiv` 91 e.V. (InteraXion / Wohnraumberatung für Geflüchtete); Place4Refugees e.V.; Willkommen im Westend; Wohnscouting Friedrichshain-Kreuzberg; Xenion AG Wohnen (2019). Gemeinsame Position der Geflüchteten-Initiativen zum Wohnberechtigungsschein. In: Fair mieten Fair wohnen. Alternativen zur öffentlichen Unterbringung Geflüchteter. Ergebnisse des Runden Tisches und der Thementische 2018/2019. Berlin: Fair mieten - Fair wohnen. Berliner Fachstelle gegen Diskriminierung auf dem Wohnungsmarkt. S. 31-35. Abgerufen am 23.03.2021 unter https://fairmieten-fairwohnen.d e/wp-content/uploads/2020/06/FMFW_Alternativen-zur-Unterbringung-Gefluec hteter.pdf

Foroutan, Naika; Hamann, Ulrike; El-Kayed, Nihad; Jorek, Susanna/ Berliner Institut für empirische Integrations- und Migrationsforschung (BIM) (2017a). Zwischen Lager und Mietvertrag: Wohnunterbringung geflüchteter Frauen in Berlin und Dresden. Berlin: Berliner Institut für empirische Integrations- und Migrationsforschung (BIM), Humboldt-Universität zu Berlin.

Foroutan, Naika; Hamann, Ulrike; El-Kayed, Nihad; Jorek, Susanna (2017b). Welchen Zugang haben Geflüchtete zum Wohnungsmarkt? Berlin: Berliner Institut für empirische Integrations- und Migrationsforschung (BIM), Humboldt Universität zu Berlin/ Mediendienst Integration.

GLADT (2021). Über uns. GLADT e.V. - unabhängig, mehrsprachig, queer. Berlin: GLADT e.V. Abgerufen am 23.03.2021 unter https://gladt.de/ueber-uns-2/

Golembe, Jasmine; Leyendecker, Birgit; Busch, Julian (2019). Psychosoziale Lage und gesellschaftliche Teilhabe von LSBTI-Geflüchteten in Deutschland: Forschungsstand und Anwendungsmöglichkeiten für die Jugendhilfe. In: Junge Geflüchtete in der Jugendhilfe: Chancen und Herausforderungen der Integration. Nowacki, Katja/ Remiorz, Silke (Hrsg.). Wiesbaden: Springer VS. S. 123-138.

Grote, Janne/ Bundesamt für Migration und Flüchtlinge (Hrsg.) (2018). Die veränderte Fluchtmigration in den Jahren 2014 bis 2016: Reaktionen und Maßnahmen in Deutschland. Fokusstudie der deutschen nationalen Kontaktstelle für das Europäische Migrationsnetzwerk (EMN). Nürnberg: Bundesamt für Migration und Flüchtlinge. Abgerufen am 28.03.2021 unter https:/www.bamf.de/Share dDocs/Anlagen/DE/EMN/Studien/wp79-emn-fluchtmigration-2014-2016-reaktio nen-ma\%C3\%9Fnahmen-deutschland.html?nn=282022 
Hassino, Mahmoud (2017). Arbeit mit Sprachmittlung. In: „Wie eine Rose, die aus dem Riss im Beton wächst." Empowerment von lesbischen, schwulen, bisexuellen sowie trans* und inter* Geflüchteten durch Beratung und Unterstützung. Berlin: Schwulenberatung Berlin. S.29-35. Abgerufen am 28.03.2021 unter https://schwulenberatungberlin.de/post/empowerment-fur-schwule-lesbische-bis exuelle-sowie-trans-und-inter-gefluchtete-eine-handreichung-fur-beratung-und-u nterstutzung

Hinger, Sophie; Schäfer, Philipp (2017)."Wohnst Du schon - oder wirst Du noch untergebracht?" Eine Bestandsaufnahme der Wohnsituation Asylsuchender in Deutschland. Bonn: Bundeszentrale für politische Bildung. Abgerufen am 07.08.2020 unter https://www.bpb.de/gesellschaft/migration/kurzdossiers/24 3947/wohnst-du-schon-oder-wirst-du-noch-untergebracht

Holm, Andrej (2016). Sozialer Wohnraumversorgungsbedarf in Berlin. Studie im Auftrag: DIE LINKE. Fraktion im Abgeordnetenhaus von Berlin. Berlin: Humboldt Universität zu Berlin. Abgerufen am 23.03.2021 unter https://www.sowi.h u-berlin.de/de/lehrbereiche/stadtsoz/mitarbeiterinnen/copy_of_a-z/holm/bericht -wohnraumversorgungsbedarf-berlin-holm-2016.pdf

Holm, Andrej; Lebuhn, Hendrik; Junker, Stephan; Neitzel, Kevin (2018). Wie viele und welche Wohnungen fehlen in deutschen Großstädten? Die soziale Versorgungslücke nach Einkommen und Wohnungsgröße. Working Paper Forschungsförderung Nummer 063. Düsseldorf: Hans-Böckler-Stiftung.

Immoscout24.de (2021). Mietendeckel-Analyse zum einjährigen Bestehen. Berlin: Immobilien Scout GmbH. Abgerufen am 19.03.2021 unter https://www.immob ilienscout24.de/unternehmen/news-medien/news/default-title/mietendeckel-anal yse-zum-einjaehrigen-bestehen/

Kleiner, Bettina; Scheunemann, Kim (2016). Trans*/ Trans*Geschlechtlichkeit. In: Gender Glossar. Leipzig: Gender Glossar. Abgerufen am 18.10.2020 unter https://gender-glossar.de/t/item/54-trans-geschlechtlichkeit

Küppers, Carolin/ Bundesstiftung Magnus Hirschfeld (Hrsg.) (2019). Refugees \& Queers: Forschung und Bildung an der Schnittstelle von LSBTTIQ, Fluchtmigration und Emanzipationspolitiken. Bielefeld: transcript.

Küppers, Carolin/ Hens, Kristina (2019). Einleitung. In: Refugees \& Queers: Forschung und Bildung an der Schnittstelle von LSBTTIQ, Fluchtmigration und Emanzipationspolitiken. Küppers, Carolin / Bundesstiftung Magnus Hirschfeld (Hrsg.). Berlin: transcript. S. 7-18.

LesMigraS (2015a). Stellungnahme zur Situation von asylsuchenden LSBTIQ in Erstaufnahmeeinrichtungen und Gemeinschaftsunterkünften in Berlin. Berlin: Lesbenberatung Berlin e.V. Abgerufen am 07.08.2020 unter http://www.lesbenb eratung-berlin.de/tl_files/lesmigras/pressemitteilungen/Stellungnahme_asylsuch ende_LSBTIQ_LesMigraS_webseite.pdf

LesMigraS (2015b). Selbstverständnis. Berlin: Lesbenberatung Berlin e.V. Abgerufen am 23.02.2021 unter https://lesmigras.de/selbstverstaendnis.html 
Nesterko, Yuriy; Jäckle, David; Friedrich, Michael; Holzapfel, Laura; Glaesmer, Heide (2020). Prevalence of post-traumatic stress disorder, depression and somatisation in recently arrived refugees in Germany: An epidemiological study. Epidemiology and Psychiatric Sciences, 29, E40. S. 1-11. Abgerufen am 23.03.2021 unter https:/www.cambridge.org/core/journals/epidemiology-and-psychiatric-sc iences/article/prevalence-of-posttraumatic-stress-disorder-depression-and-somatis ation-in-recently-arrived-refugees-in-germany-an-epidemiological-study/172350E F44E4E8F477B237365C922842

nonbinary.ch (2021). Was ist non-binäres Geschlecht? Adliswil: nonbinary.ch. Abgerufen am 18.03.2021 unter https:/www.nonbinary.ch/was-ist-non-binaer/

Pieper, Tobias (2013). Die Gegenwart der Lager: Zur Mikrophysik der Herrschaft in der deutschen Flüchtlingspolitik. 2. Auflage. Münster: Westfälisches Dampfboot.

Queer Lexikon (2020a). Passing. Freiburg: Queer Lexikon e.V. Abgerufen am 16.10.2020 unter: https:/queer-lexikon.net/2017/06/08/passing/

Queer Lexikon (2020b). Dyadisch. Freiburg: Queer Lexikon e.V. Abgerufen am 18.03.2021 unter https:/queer-lexikon.net/2017/06/15/dyadik/

Queer Lexikon (2020c). Transmisogynie. Freiburg: Queer Lexikon e.V. Abgerufen am 23.03.2021 unter https:/queer-lexikon.net/2020/04/29/trans-misogynie/

Queer Lexikon (2021): Inter. Freiburg: Queer Lexikon e.V. Abgerufen am 18.03.2021 unter https:/queer-lexikon.net/2017/06/08/inter/

Rittinghaus, Gesa L. (2017). Ehrenamtsmanagement im LSBT*T*I-Bereich. In: „Wie eine Rose, die aus dem Riss im Beton wächst." Empowerment von lesbischen, schwulen, bisexuellen sowie trans* und inter* Geflüchteten durch Beratung und Unterstützung. Berlin: Schwulenberatung Berlin. S. 25-28. Abgerufen am 28.03.2021 unter https://schwulenberatungberlin.de/post/empowerment-furschwule-lesbische-bisexuelle-sowie-trans-und-inter-gefluchtete-eine-handreichun g-fur-beratung-und-unterstutzung

Sauer, Madeleine; Vey, Judith (2019). Sozialräumliche Integration von Geflüchteten im ländlichen Raum. In: Wissen schafft Demokratie (5)2019. Berlin: Amadeu Antonio Stiftung. S. 52-61. Abgerufen am 18.03. 2021 unter https://www.idz -jena.de/wsddet/wsd5-9-1/

Schwulenberatung (2020a). Wir helfen. Berlin: Schwulenberatung Berlin. Abgerufen am 22.03.2021 unter https://schwulenberatungberlin.de/

Schwulenberatung (2020b). Queer Refugees. Berlin: Schwulenberatung Berlin. Abgerufen am 22.03.2021 unter https://schwulenberatungberlin.de/queer-refugees/ queer-refugees-de

Senatsverwaltung für Integration, Arbeit und Soziales (2010). Deutscher Name halbe Miete? Diskriminierung auf dem Wohnungsmarkt. Berlin: Senatsverwaltung für Integration, Arbeit und Soziales. Landesstelle für Gleichbehandlung gegen Diskriminierung. Abgerufen am 23.03.2021 unter https:/www.berlin.de/s en/lads/_assets/ueber-uns/materialien/deutscher_name-halbe_miete_bf.pdf 
Senatsverwaltung für Integration, Arbeit und Soziales (2020). Abschlussveranstaltung Runder Tisch Wohnen für Geflüchtete als Videokonferenz. Pressemitteilung vom 03.09.2020. Berlin: Senatsverwaltung für Integration, Arbeit und Soziales. Abgerufen am 30.01.2021 unter https://www.berlin.de/sen/ias/press e/pressemitteilungen/2020/pressemitteilung.986026.php

Senat von Berlin (2015): Versorgungs- und Integrationskonzept für Asylbegehrende und Flüchtlinge. Berlin: Senat von Berlin. Abgerufen am 18.10.2020 unter https://fluechtlingsrat-berlin.de/wp-content/uploads/FluechtlingskonzeptSenat.p df

Shevtsova, Maryna (2019). (Mis-) Representing LGBTI Refugees. Instrumentalisation Strategies in Media Coverage of Asylum Policy in Germany. In: Refugees \& Queers: Forschung und Bildung an der Schnittstelle von LSBTTIQ, Fluchtmigration und Emanzipationspolitiken. Küppers, Carolin / Bundesstiftung Magnus Hirschfeld (Hrsg.). Berlin: transcript. S. 83-99.

Tanyılmaz, Tuğba; Ünsal, Nadiye; Yılmaz-Günay, Koray (2019). Ein Leben, das für alle lebbar ist? Schutzsuchende LSBTTIQ zwischen Mainstreamisierung und Exzeptionalismus. In: Refugees \& Queers: Forschung und Bildung an der Schnittstelle von LSBTTIQ, Fluchtmigration und Emanzipationspolitiken. Küppers, Carolin / Bundesstiftung Magnus Hirschfeld (Hrsg.). Berlin: transcript. S. 137-152.

Täubig, Vicki (2009). Totale Institution Asyl: Empirische Befunde zu alltäglichen Lebensführungen in der organisierten Desintegration. München: Juventa.

Wendel, Kay/ Pro Asyl (Hrsg.) (2014). Unterbringung von Flüchtlingen in Deutschland: Regelungen und Praxis der Bundesländer im Vergleich. Frankfurt am Main: Pro Asyl. Abgerufen am 07.08.2020 unter https://www.proasyl.de/mat erial/studie-zur-unterbringung-von-fluechtlingen-in-deutschland/

Wendel, Kay/ Antidiskriminierungsberatung Brandenburg (2016). Hintergrundinformation Juli 2016: Unter welchen aufenthaltsrechtlichen Bedingungen haben Flüchtlinge einen rechtlichen Anspruch auf Umzug in eine eigene Wohnung? Potsdam: Antidiskriminierungsberatung Brandenburg. Abgerufen am 16.10.2020 unter https://www.antidiskriminierungsberatung-brandenburg.d e/wp-content/uploads/2019/04/Hintergrundinformation_Juli-2016_Recht-auf-A uszug_ADB_2.pdf 
